\title{
Dual Boundary Method for Assembled Plate Structures Undergoing Large Deflection
}

\author{
C.Di Pisa ${ }^{1}$, M.H. Aliabadi ${ }^{2}$ and A.Young ${ }^{3}$ \\ ${ }^{1}$ Department of Engineering, Queen Mary, University of London, London E1 4NS, UK \\ ${ }^{2}$ Department of Aeronautics, Imperial College, London SW7 2BY, UK \\ ${ }^{3}$ Future Systems Technology, Materials QinetQ, Farnborough, Hants GU14 0LX, UK
}

\begin{abstract}
In this paper, the Dual Boundary Element Method is combined with a multi-region formulation to simulate plate assembly undergoing large deflection. The incremental load approach is used to treat the geometrical non-linearity and Radial Basis Functions are used to approximate the derivatives of the large deflection terms. The Dual Reciprocity Method is used to transfer to the boundary all the domain integrals. Once the solution at the boundary is obtained for the assembly, a J-integral for large deflection is implemented to extract the fracture parameters.
\end{abstract}

Keywords: Dual Boundary Method; Reissner plate; Large Deflection; Radial Basis Functions; Assembled plate structures

\section{Introduction}

Thin plate structures such as those employed in aircraft parts, may undergo large deflection with normal service loads. In order to investigate such case, the assembled plate formulation proposed by Dirgantara and Aliabadi [11][10], is extended to plate structures undergoing large deflection. The shear deformable plate theory developed by Reissner [8] in 1945, is used to describe the bending behaviour, while 2D elasticity is used for the membrane. The two formulations are presented together to simulate the full plate behaviour as membrane and bending are coupled in large deflection theory. Von Karman's approach [4] is followed to describe the interaction between membrane and bending of the plate. The Dual Reciprocity Method (DRM) is implemented to transfer to the boundary the domain integrals of the large deflection contribution, as shown in [17]. Boundary element application to large deflection theory for plates can be found in [7], [9] and [6].

The Dual Boundary Element Method (DBEM) formulated for shear deformable plates by Dirgantara and Aliabadi in [14] is extended to large deflection analysis and then is combined with the multi-region formulation to simulate cracks in plate assembly undergoing large deflection. In shear deformable plate theory, the stress and displacement fields near the crack tip cannot be described by the standard stress intensity factors (SIFs) as higher order terms need to be included. The Crack Opening Displacement (COD) cannot be applied to such cases, hence a J-integral formulation comprehensive of large deflection contributions is proposed to extract the fracture parameters.

\section{Boundary integral equations for large deflection}

The equilibrium equations for shear deformable plates undergoing large deflection can be written as follows [17]:

$$
\begin{gathered}
\hat{N}_{\alpha \beta, \beta}+\left[\dot{N}_{\alpha \beta, \beta}+f_{\alpha}\right]=0 \\
M_{\alpha \beta, \beta}-Q_{\alpha}+q_{\alpha}=0 \\
Q_{\alpha, \alpha}+\left[\left(N_{\alpha \beta} w_{3, \beta}\right)_{, \alpha}+q_{3}\right]=0
\end{gathered}
$$


where the membrane stress resultants have been divided into components related to in-plane displacements $\left(\hat{N}_{\alpha \beta, \beta}\right)$ and to deflection $\left(\dot{N}_{\alpha \beta, \beta}\right)$, as follows:

$$
\begin{gathered}
\hat{N}_{\alpha \beta}=B \frac{1-\nu}{2}\left(u_{\alpha, \beta}+u_{\beta, \alpha}+\frac{2 \nu}{1-\nu} u_{\gamma, \gamma} \delta_{\alpha, \beta}\right) \\
\dot{N}_{\alpha \beta}=\frac{B}{2} w_{3, \alpha} w_{3, \beta}
\end{gathered}
$$

The terms in brackets [ ] in (1) are grouped and considered as domain loads and the equilibrium equations assume the same form as for small deflection; thus the boundary integral equation can derived in the same way and they can be written as follows:

$$
\begin{gathered}
C_{\alpha \beta}^{m}\left(\mathbf{x}^{\prime}\right) u_{\beta}\left(\mathbf{x}^{\prime}\right)+\int_{\Gamma} T_{\alpha \beta}\left(\mathbf{x}^{\prime}, \mathbf{x}\right) u_{\beta}(\mathbf{x}) d \Gamma-\int_{\Gamma} U_{\alpha \beta}\left(\mathbf{x}^{\prime}, \mathbf{x}\right) \hat{t}_{\beta}(\mathbf{x}) d \Gamma= \\
\int_{\Omega} U_{\alpha \beta}\left(\mathbf{x}^{\prime}, \mathbf{X}\right)\left[\dot{N}_{\beta \gamma, \gamma}(\boldsymbol{X})+f_{\beta}(\boldsymbol{X})\right] d \Omega \\
C_{i k}^{b}\left(\mathbf{x}^{\prime}\right) w_{k}\left(\mathbf{x}^{\prime}\right)+\int_{\Gamma} P_{i k}\left(\mathbf{x}^{\prime}, \mathbf{x}\right) w_{k}(\mathbf{x}) d \Gamma-\int_{\Gamma} W_{i k}\left(\mathbf{x}^{\prime}, \mathbf{x}\right) p_{k}(\mathbf{x}) d \Gamma= \\
\int_{\Omega} W_{i k}\left(\mathbf{x}^{\prime}, \mathbf{X}\right)\left[\delta_{3 k}\left(N_{\alpha \beta} w_{3, \beta}\right)_{, \alpha}(\boldsymbol{X})+q_{k}(\boldsymbol{X})\right] d \Omega
\end{gathered}
$$

where the kernels $T_{\alpha \beta}\left(\mathbf{x}^{\prime}, \mathbf{x}\right), U_{\alpha \beta}\left(\mathbf{x}^{\prime}, \mathbf{x}\right), P_{i k}\left(\mathbf{x}^{\prime}, \mathbf{x}\right)$ and $W_{i k}\left(\mathbf{x}^{\prime}, \mathbf{x}\right)$ are the same fundamental solutions as for small deflection and can be found in Appendix A. The second boundary integral in equation (3) is expressed in terms of boundary tractions $\hat{t}_{\beta}$, which are related to the $\hat{N}_{\alpha \beta}$ stress resultants as follows:

$$
\hat{N}_{\alpha \beta} n_{\beta}=\hat{t}_{\beta}
$$

In order to enforce the equilibrium conditions at join-lines, it is necessary to express (3) in terms of the real boundary traction $t_{\beta}$. Considering the following relation:

$$
t_{\beta}=N_{\alpha \beta} n_{\beta}=\hat{N}_{\alpha \beta} n_{\beta}+\dot{N}_{\alpha \beta} n_{\beta}=\hat{t}_{\beta}+\dot{t}_{\beta}
$$

and substituting into equation (3), it is obtained:

$$
\begin{gathered}
C_{\alpha \beta}^{m}\left(\mathbf{x}^{\prime}\right) u_{\beta}\left(\mathbf{x}^{\prime}\right)+\int_{\Gamma} T_{\alpha \beta}\left(\mathbf{x}^{\prime}, \mathbf{x}\right) u_{\beta}(\mathbf{x}) d \Gamma-\int_{\Gamma} U_{\alpha \beta}\left(\mathbf{x}^{\prime}, \mathbf{x}\right) t_{\beta}(\mathbf{x}) d \Gamma= \\
\int_{\Omega} U_{\alpha \beta}\left(\mathbf{x}^{\prime}, \mathbf{X}\right)\left[\dot{N}_{\beta \gamma, \gamma}(\mathbf{X})+f_{\beta}(\mathbf{X})\right] d \Omega-\int_{\Gamma} U_{\alpha \beta}\left(\mathbf{x}^{\prime}, \mathbf{x}\right) \dot{t}_{\beta}(\mathbf{x}) d \Gamma
\end{gathered}
$$

where $\dot{t}_{\beta}=\dot{N}_{\alpha \beta} n_{\beta}$.

The above equations, together with equations (4), form a complete set of boundary integral equations for the elastic plate in large deflection.

\section{Internal stress resultants}

The stress resultants $N_{\alpha \beta}\left(\mathbf{X}^{\prime}\right)$ at domain point $\left(\mathbf{X}^{\prime}\right)$ can be written as sum of $\hat{N}_{\alpha \beta}\left(\mathbf{X}^{\prime}\right)$ and $\dot{N}_{\alpha \beta}\left(\mathbf{X}^{\prime}\right)$ as follows:

$$
N_{\alpha \beta}\left(\mathbf{X}^{\prime}\right)=\hat{N}_{\alpha \beta}\left(\mathbf{X}^{\prime}\right)+\dot{N}_{\alpha \beta}\left(\mathbf{X}^{\prime}\right)
$$

where $\hat{N}_{\alpha \beta}\left(\mathbf{X}^{\prime}\right)$ and $\dot{N}_{\alpha \beta}\left(\mathbf{X}^{\prime}\right)$ have to be derived separately. 
The first term $\hat{N}_{\alpha \beta}\left(\mathbf{X}^{\prime}\right)$, can be written as follows:

$$
\begin{aligned}
\hat{N}_{\alpha \beta}\left(\mathbf{X}^{\prime}\right) & =\int_{\Gamma} D_{\alpha \beta \gamma}^{m}\left(\mathbf{X}^{\prime}, \mathbf{x}\right) \hat{t}_{\gamma}(\mathbf{x}) d \Gamma-\int_{\Gamma} S_{\alpha \beta \gamma}^{m}\left(\mathbf{X}^{\prime}, \mathbf{x}\right) u_{\gamma}(\mathbf{x}) d \Gamma+ \\
& +\int_{\Omega} D_{\alpha \beta \gamma}^{m}\left(\mathbf{X}^{\prime}, \mathbf{X}\right)\left[\dot{N}_{\beta \gamma, \gamma}(\mathbf{X})+f_{\gamma}(\mathbf{X})\right] d \Omega
\end{aligned}
$$

where $D_{\alpha \beta \gamma}^{m}$ and $S_{\alpha \beta \gamma}^{m}$ are linear combination of fundamental solutions and are given in Appendix A.

Writing the stress resultants $N_{\alpha \beta}\left(\mathbf{X}^{\prime}\right)$ in term of traction $t_{\gamma}$ and recalling that $\dot{N}_{\alpha \beta}\left(\mathbf{X}^{\prime}\right)=\frac{B}{2} w_{3, \alpha} w_{3, \beta}$, it is obtained:

$$
\begin{gathered}
N_{\alpha \beta}\left(\mathbf{X}^{\prime}\right)=\int_{\Gamma} D_{\alpha \beta \gamma}^{m}\left(\mathbf{X}^{\prime}, \mathbf{x}\right) t_{\gamma}(\mathbf{x}) d \Gamma-\int_{\Gamma} S_{\alpha \beta \gamma}^{m}\left(\mathbf{X}^{\prime}, \mathbf{x}\right) u_{\gamma}(\mathbf{x}) d \Gamma+ \\
+\int_{\Omega} D_{\alpha \beta \gamma}^{m}\left(\mathbf{X}^{\prime}, \mathbf{X}\right)\left[\dot{N}_{\beta \gamma, \gamma}(\mathbf{X})+f_{\gamma}(\mathbf{X})\right] d \Omega-\int_{\Gamma} D_{\alpha \beta \gamma}^{m}\left(\mathbf{X}^{\prime}, \mathbf{x}\right) \dot{t}_{\gamma}(\mathbf{x}) d \Gamma+\frac{B}{2} w_{3, \alpha} w_{3, \beta}
\end{gathered}
$$

The shear and moment stress resultants at domain point $\left(\mathbf{X}^{\prime}\right)$ can be obtained in the same way as for small deflection, as their definition has not changed:

$$
\begin{aligned}
M_{\alpha \beta}\left(\mathbf{X}^{\prime}\right) & =\int_{\Gamma} D_{\alpha \beta \gamma}^{b}\left(\mathbf{X}^{\prime}, \mathbf{x}\right) p_{\gamma}(\mathbf{x}) d \Gamma-\int_{\Gamma} S_{\alpha \beta \gamma}^{b}\left(\mathbf{X}^{\prime}, \mathbf{x}\right) w_{\gamma}(\mathbf{x}) d \Gamma+ \\
& +\int_{\Omega} D_{\alpha \beta \gamma}^{b}\left(\mathbf{X}^{\prime}, \mathbf{X}\right) q_{\gamma}(\mathbf{X}) d \Omega \\
Q_{\beta}\left(\mathbf{X}^{\prime}\right) & =\int_{\Gamma} D_{3 \beta \gamma}^{b}\left(\mathbf{X}^{\prime}, \mathbf{x}\right) p_{\gamma}(\mathbf{x}) d \Gamma-\int_{\Gamma} S_{3 \beta \gamma}^{b}\left(\mathbf{X}^{\prime}, \mathbf{x}\right) w_{\gamma}(\mathbf{x}) d \Gamma+ \\
+ & \int_{\Omega} D_{3 \beta 3}^{b}\left(\mathbf{X}^{\prime}, \mathbf{X}\right)\left[\left(N_{\alpha \beta} w_{3, \beta}\right)_{, \alpha}(\mathbf{X})+q_{3}(\mathbf{X})\right] d \Omega
\end{aligned}
$$

where $D_{\alpha \beta \gamma}^{b}, S_{\alpha \beta \gamma}^{b}, D_{3 \beta \gamma}^{b}$ and $S_{3 \beta \gamma}^{b}$ are the same fundamental solutions derived for small deflection and are given in Appendix A. In the domain integral of the shear stress resultants $\left(Q_{\beta}\right)$, it has to be noticed the presence of the term $\left(N_{\alpha \beta} w_{3, \beta}\right)_{, \alpha}$, which is considered as an additional domain load.

\section{Dual boundary integral equations in large deflection}

Consider a cracked plate where $\Gamma$ is the external boundary and $\Gamma^{+}, \Gamma^{-}$are the boundaries of the crack. Before deformation takes place, the boundaries $\Gamma^{+}$and $\Gamma^{-}$are coincident. If boundary integral displacement equations are applied on both boundaries, they result identical as shown in [1]. Another set of independent equations needs to be employed in order to build a non-singular system. Following [2] this extra set is provided by the boundary traction integral equations; these equations are found to be independent from the boundary displacement integral equations and can be applied to either of the crack boundaries. The boundary traction integral equations can be derived by considering the equations that relate the stress resultants and the boundary tractions. By taking the integral expressions of the stress resultants (equations (10a), (10b) and (10c)) to the boundary $\left(\left(\mathbf{X}^{\prime} \in \Omega\right) \longrightarrow\left(\mathbf{x}_{-}^{\prime} \in \Gamma^{-}\right)\right)$, considering that $\left(n_{\beta}\left(\mathbf{x}^{\prime} \in \Gamma^{+}\right)=-n_{\beta}\left(\mathbf{x}^{\prime} \in \Gamma^{-}\right)\right)$and assuming that $\Gamma^{-}$ is smooth, they can be written as follows:

$$
\begin{gathered}
\frac{1}{2} N_{\alpha \beta}\left(\mathbf{x}_{-}^{\prime}\right)-\frac{1}{2} N_{\alpha \beta}\left(\mathbf{x}_{+}^{\prime}\right)=f_{\Gamma} D_{\alpha \beta \gamma}^{m}\left(\mathbf{x}_{-}^{\prime}, \mathbf{x}\right) t_{\gamma}(\mathbf{x}) d \Gamma-\int_{\Gamma} S_{\alpha \beta \gamma}^{m}\left(\mathbf{x}_{-}^{\prime}, \mathbf{x}\right) u_{\gamma}(\mathbf{x}) d \Gamma+ \\
-f_{\Gamma} D_{\alpha \beta \gamma}^{m}\left(\mathbf{x}_{-}^{\prime}, \mathbf{x}\right) \dot{t}_{\gamma}(\mathbf{x}) d \Gamma+\int_{\Omega} D_{\alpha \beta \gamma}^{m}\left(\mathbf{x}_{-}^{\prime}, \mathbf{X}\right)\left[\dot{N}_{\beta \gamma, \gamma}(\mathbf{X})+f_{\gamma}(\mathbf{X})\right] d \Omega
\end{gathered}
$$


for plate membrane, and for plate bending as:

$$
\begin{aligned}
& \frac{1}{2} M_{\alpha \beta}\left(\mathbf{x}_{-}^{\prime}\right)-\frac{1}{2} M_{\alpha \beta}\left(\mathbf{x}_{+}^{\prime}\right)=f_{\Gamma} D_{\alpha \beta k}^{b}\left(\mathbf{x}_{-}^{\prime}, \mathbf{x}\right) p_{k}(\mathbf{x}) d \Gamma-\int_{\Gamma} S_{\alpha \beta k}^{b}\left(\mathbf{x}_{-}^{\prime}, \mathbf{x}\right) w_{k}(\mathbf{x}) d \Gamma+ \\
& +\int_{\Omega} D_{\alpha \beta \gamma}^{b}\left(\mathbf{x}_{-}^{\prime}, \mathbf{X}\right) q_{\gamma}(\mathbf{X}) d \Omega+\int_{\Omega} D_{\alpha \beta 3}^{b}\left(\mathbf{x}_{-}^{\prime}, \mathbf{X}\right)\left[\left(N_{\alpha \beta} w_{3, \beta}\right)_{, \alpha}(\mathbf{X})+q_{3}(\mathbf{X})\right] d \Omega \\
& \quad \frac{1}{2} Q_{\beta}\left(\mathbf{x}_{-}^{\prime}\right)-\frac{1}{2} Q_{\beta}\left(\mathbf{x}_{+}^{\prime}\right)=f_{\Gamma} D_{3 \beta k}^{b}\left(\mathbf{x}_{-}^{\prime}, \mathbf{x}\right) p_{k}(\mathbf{x}) d \Gamma-\int_{\Gamma} S_{3 \beta k}^{b}\left(\mathbf{x}_{-}^{\prime}, \mathbf{x}\right) w_{k}(\mathbf{x}) d \Gamma+ \\
& \quad+\int_{\Omega} D_{3 \beta \gamma}^{b}\left(\mathbf{x}_{-}^{\prime}, \mathbf{X}\right) q_{\gamma}(\mathbf{X}) d \Omega+\int_{\Omega} D_{3 \beta 3}^{b}\left(\mathbf{x}_{-}^{\prime}, \mathbf{X}\right)\left[\left(N_{\alpha \beta} w_{3, \beta}\right)_{, \alpha}(\mathbf{X})+q_{3}(\mathbf{X})\right] d \Omega
\end{aligned}
$$

where the symbol $f$ stands for Hadamard principal value integral.

Introducing equations (11a), (11b) and (11c) into the relation between stress resultants and boundary tractions, the boundary traction integral equations in large deflection are obtained as follows:

$$
\begin{gathered}
\frac{1}{2} t_{\alpha}\left(\mathbf{x}_{-}^{\prime}\right)-\frac{1}{2} t_{\alpha}\left(\mathbf{x}_{+}^{\prime}\right)=n_{\beta}\left(\mathbf{x}_{-}^{\prime}\right) f_{\Gamma} D_{\alpha \beta \gamma}^{m}\left(\mathbf{x}_{-}^{\prime}, \mathbf{x}\right) t_{\gamma}(\mathbf{x}) d \Gamma-n_{\beta} \int_{\Gamma} S_{\alpha \beta \gamma}^{m}\left(\mathbf{x}_{-}^{\prime}, \mathbf{x}\right) u_{\gamma}(\mathbf{x}) d \Gamma+ \\
-n_{\beta} f_{\Gamma} D_{\alpha \beta \gamma}^{m}\left(\mathbf{x}_{-}^{\prime}, \mathbf{x}\right) \dot{t}_{\gamma}(\mathbf{x}) d \Gamma+n_{\beta} \int_{\Omega} D_{\alpha \beta \gamma}^{m}\left(\mathbf{x}_{-}^{\prime}, \mathbf{X}\right)\left[\dot{N}_{\beta \gamma, \gamma}(\mathbf{X})+f_{\gamma}(\mathbf{X})\right] d \Omega
\end{gathered}
$$

for plate membrane, and

$$
\begin{gathered}
\frac{1}{2} p_{\alpha}\left(\mathbf{x}_{-}^{\prime}\right)-\frac{1}{2} p_{\alpha}\left(\mathbf{x}_{-}^{\prime}\right)=n_{\beta}\left(\mathbf{x}_{-}^{\prime}\right) f_{\Gamma} D_{\alpha \beta k}^{b}\left(\mathbf{x}_{-}^{\prime}, \mathbf{x}\right) p_{k}(\mathbf{x}) d \Gamma-n_{\beta} \int_{\Gamma} S_{\alpha \beta k}^{b}\left(\mathbf{x}_{-}^{\prime}, \mathbf{x}\right) w_{k}(\mathbf{x}) d \Gamma+ \\
+n_{\beta} \int_{\Omega} D_{\alpha \beta \gamma}^{b}\left(\mathbf{x}_{-}^{\prime}, \mathbf{X}\right) q_{\gamma}(\mathbf{X}) d \Omega+\int_{\Omega} D_{\alpha \beta 3}^{b}\left(\mathbf{x}_{-}^{\prime}, \mathbf{X}\right)\left[\left(N_{\alpha \beta} w_{3, \beta}\right)_{, \alpha}(\mathbf{X})+q_{3}(\mathbf{X})\right] d \Omega \\
\frac{1}{2} p_{3}\left(\mathbf{x}_{-}^{\prime}\right)-\frac{1}{2} p_{3}\left(\mathbf{x}_{-}^{\prime}\right)=n_{\beta}\left(\mathbf{x}_{-}^{\prime}\right) f_{\Gamma} D_{3 \beta k}^{b}\left(\mathbf{x}_{-}^{\prime}, \mathbf{x}\right) p_{k}(\mathbf{x}) d \Gamma-n_{\beta} \int_{\Gamma} S_{3 \beta k}^{b}\left(\mathbf{x}_{-}^{\prime}, \mathbf{x}\right) w_{k}(\mathbf{x}) d \Gamma+ \\
\quad+n_{\beta} \int_{\Omega} D_{3 \beta \gamma}^{b}\left(\mathbf{x}_{-}^{\prime}, \mathbf{X}\right) q_{\gamma}(\mathbf{X}) d \Omega+n_{\beta} \int_{\Omega} D_{3 \beta k}^{b}\left(\mathbf{x}_{-}^{\prime}, \mathbf{X}\right)\left[\left(N_{\alpha \beta} w_{3, \beta}\right)_{, \alpha}(\mathbf{X})+q_{3}(\mathbf{X})\right] d \Omega
\end{gathered}
$$

for plate bending.

It is worth noticing that the large deflection tractions $\left(\dot{t}_{\gamma}\right)$ referred to the crack boundaries points cancel each-other as $n_{\beta}\left(\mathbf{x}^{\prime} \in \Gamma^{+}\right)=-n_{\beta}\left(\mathbf{x}^{\prime} \in \Gamma^{-}\right)$in the integral $n_{\beta} f \frac{f_{\Gamma}}{D_{\alpha \beta \gamma}^{m}}\left(\mathbf{x}^{\prime}, \mathbf{x}\right) \dot{t}_{\gamma}(\mathbf{x}) d \Gamma$.

\section{Multi-region formulation for modelling assembled plate structures}

In order to model assembled plate structures, a multi-region formulation is implemented similarly as that proposed by Dirgantara in ([11]). Consider $M$ plates joined along a common edge (figure (1)). For each plate it is possible to write the boundary integral equation independently, in local coordinates $x_{i}^{m}$, where $i=1,2,3$ and $m=1, . ., M$. Then compatibility equations for displacements and equilibrium equations along the joining line can be enforced. Different cases have to be considered, according to the geometry.

In the simple case of two plates with same axis orientation, joined along a generic line with eccentricity $e$, the equations expressing continuity of displacements and equilibrium of stresses across the join can be written as follows: 


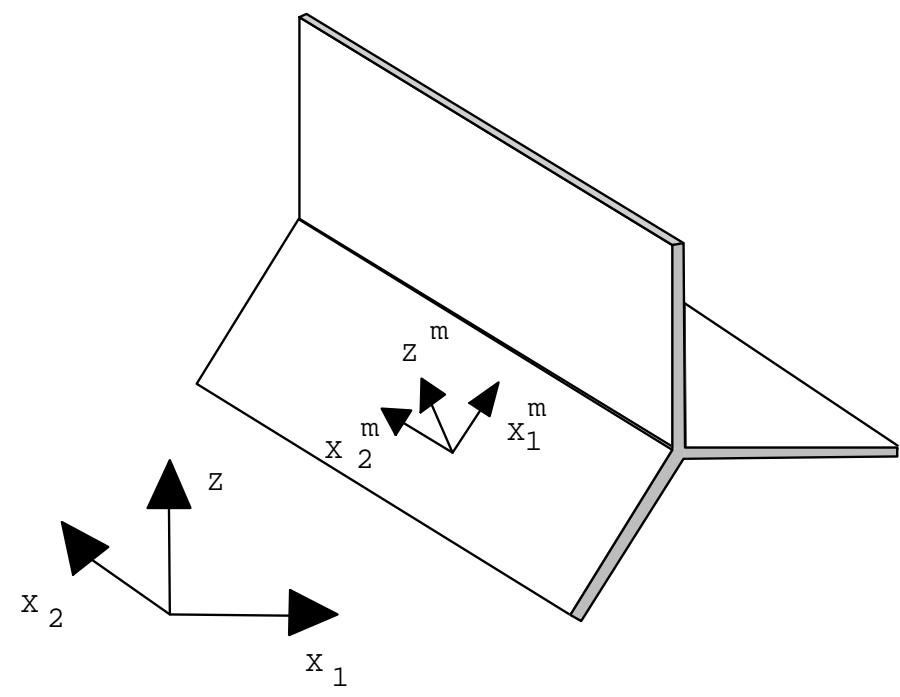

Figure 1: Generic assembled plates

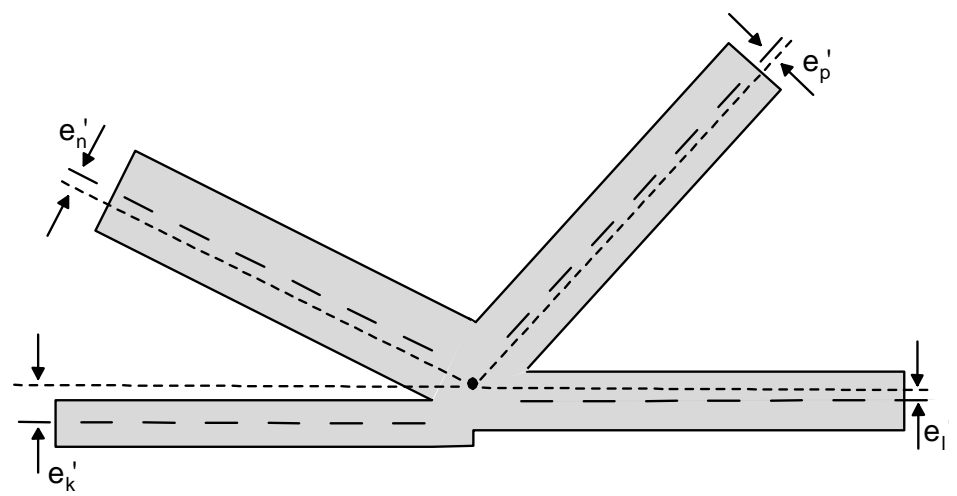

Figure 2: Off-set assembled plates with eccentricity

$$
\begin{gathered}
u_{\alpha}^{1}-e w_{\alpha}^{1}=u_{\alpha}^{2} \\
w_{i}^{1}=w_{i}^{2}
\end{gathered}
$$

for displacement compatibility and

$$
\begin{aligned}
t_{\alpha}^{1}+t_{\alpha}^{2} & =0 \\
p_{3}^{1}+p_{3}^{2} & =0 \\
p_{\alpha}^{1}-e t_{\alpha}^{1}+p_{\alpha}^{2} & =0
\end{aligned}
$$

for equilibrium at the join line.

If there are two or more angled plates joined together (figure (2)), the approach proposed in [16] is followed. In order to simplify the description, the local coordinate systems for the $M$ plates are assumed to be defined such that the $x_{2}^{m}$ directions are all aligned with the same global direction along a straight join line. 
The compatibility/equilibrium equations required along the join line are generated as follows. Each pair of plates (e.g. $m=1$ and $m=2)$ deform compatibly: for each pair $(M=2)$ of angled plates joined together, the compatibility conditions can be enforced as follows:

$$
\begin{aligned}
\left(u_{1}^{1}-e_{m}^{\prime} w_{1}^{1}\right) n_{11}^{1}+w_{3}^{1} n_{13}^{1} & =\left(u_{1}^{2}-e_{k}^{\prime} w_{1}^{2}\right) n_{11}^{2}+w_{3}^{2} n_{13}^{2} \\
\left(u_{1}^{1}-e_{m}^{\prime} w_{1}^{1}\right) n_{31}^{1}+w_{3}^{1} n_{33}^{1} & =\left(u_{1}^{2}-e_{k}^{\prime} w_{1}^{2}\right) n_{31}^{2}+w_{3}^{2} n_{33}^{2} \\
u_{2}^{1} & =u_{2}^{2} \\
w_{1}^{1} & =w_{1}^{2} \\
w_{2}^{1} & =0 \\
w_{2}^{2} & =0
\end{aligned}
$$

where $n_{i k}^{m}$ are the components of the rotation matrix of plate $m$ from local to global coordinates. For $M$ plates, equations (15) give $5 M-4$ compatibility conditions, which are supplemented by 4 equilibrium conditions:

$$
\begin{gathered}
\sum_{m=1}^{M}\left\{t_{1}^{m} n_{11}^{m}+p_{3}^{m} n_{13}^{m}\right\}=0 \\
\sum_{m=1}^{M}\left(t_{1}^{m} n_{31}^{m}+p_{3}^{m} n_{33}^{m}\right)=0 \\
\sum_{m=1}^{M} t_{2}^{m}=0 \\
\sum_{m=1}^{M}\left(p_{1}^{m}-e_{m}^{\prime} t_{1}^{m}\right)=0
\end{gathered}
$$

where $e_{m}^{\prime}$ is the eccentricity of the $m$-plate respect to the centre of the joint. What is important is the relative eccentricity, therefore the reference point can be chosen arbitrarily. Usually, the quantities $e_{m}^{\prime}$, and consequently the products $e_{m}^{\prime} t_{1}^{m}$ are small compared to $p_{1}^{m}$, and for three or more angled plates can be ignored.

\section{Numerical implementation}

For the numerical implementation, it is necessary to set an iterative procedure to linearize the non-linear equations. As explained in the following paragraphs, an incremental load approach is used and the domain term relative to large deflections are evaluated at previous steps. In this way, all the domain loads are know at each integration step and the domain integrals can be transferred to the boundary using the Dual Reciprocity Method. The additional boundary integrals due to tractions $\dot{t}$, are evaluated at each step from previous steps results, approximating the boundary tractions $\dot{t}$ with Radial Basis Functions.

\section{Iterative procedure}

The numerical procedure to solve the system of equations has been chosen considering the results obtained in [6], [9] and [17]; the system of non-linear equations generated is solved using an incremental load approach. Following [17] the load is divided into several quasi-linear steps, in which the new integrals containing the large deflection terms are considered as an additional load to be computed from the previous step. The general scheme is presented in figure (3). Several methods have been analyzed, and for this type of analysis, in the range of deflection considered (large but not too large) the total load approach with no iteration has been found to be the best in terms of accuracy and efficiency. The large deflection contributions for the $s$-step are evaluated from the $s-1$ step; for the initial step $(s=1)$, are considered equal to zero. 


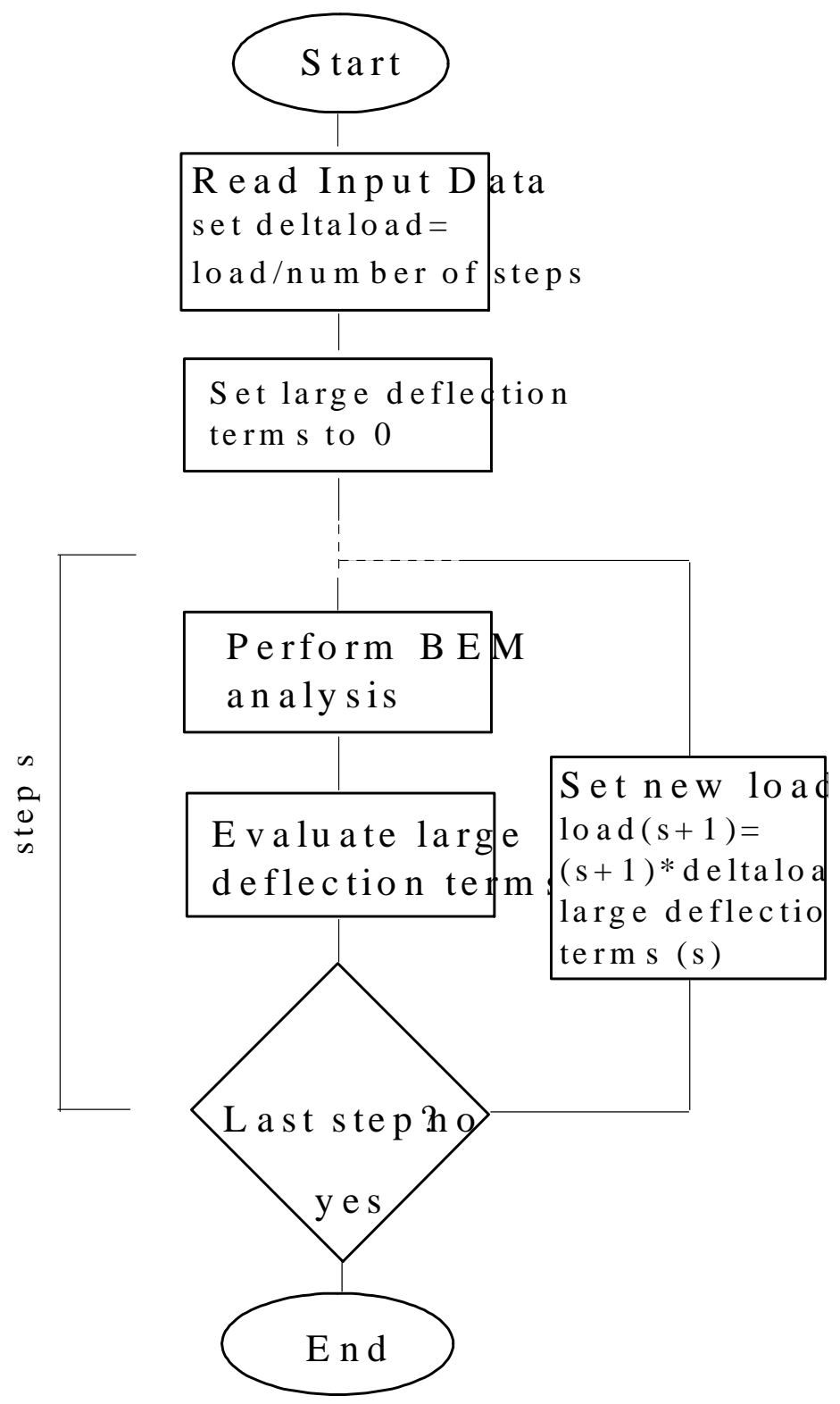

Figure 3: Flow chart of total load approach 


\section{Large deflection terms}

There are five large deflection contributions to be evaluated, two on the boundary $\left(\dot{t}_{1}, \dot{t}_{2}\right)$ and three in the domain $\left(\dot{q}_{3}, \dot{f}_{1}, \dot{f}_{2}\right)$. The latter, at a domain point $\left(\mathbf{X}^{\prime}\right)$, can be expressed in terms of deflection derivatives and normal stress resultants as follows:

$$
\begin{aligned}
\dot{q}_{3}\left(\mathbf{X}^{\prime}\right) & =\left(N_{\alpha \beta} w_{3, \beta}\right)_{, \alpha} \\
\dot{f}_{\alpha}\left(\mathbf{X}^{\prime}\right) & =\dot{N}_{\alpha \beta, \beta}=\frac{B}{2}\left(w_{3, \alpha} w_{3, \beta}\right)_{, \beta}
\end{aligned}
$$

The derivatives of the deflection $w_{3, \alpha}$ at domain points, can be written as follows:

$$
w_{3, \alpha}=\int_{\Gamma} W_{3 k, \alpha} p_{k} d \Gamma-\int_{\Gamma} P_{3 k, \alpha} w_{k} d \Gamma+\int_{\Omega} W_{3 k, \alpha}\left[q_{k}+\left(N_{\alpha \beta} w_{3, \beta}\right)_{, \alpha}\right] d \Omega
$$

where the derivatives of the kernels $\left(W_{3 k, \alpha}, P_{3 k, \alpha}\right)$ are presented in Appendix A. It has to be noticed that all the quantities are known at this point, as $w_{3, \alpha}$ is evaluated at the step $s-1$ to generate the wanted contribution to the current step $s$.

Deflection derivatives at the boundary need to be computed. It is possible to do so by taking (17) to the boundary, but a strong singularity $\left(O\left(1 / r^{2}\right)\right)$ is produced in kernels $\left(P_{3 k, \alpha}\right)$. It is possible to integrate this type of singularity, using discontinuous elements as shown for traction integral equations in [1], but it was found not to be efficient; the boundary of each plate would need to be discretized in such manner and the computational time required for computing all the corresponding kernels can be quite large. Another way to evaluate those derivatives on the boundary is to extrapolate them from the known deflections derivatives in the domain. The deflection derivatives at a boundary point $\left(\mathbf{x}^{\prime}\right)$ can be approximated with RBF, as follows:

$$
w_{3, \alpha}\left(\mathbf{x}^{\prime}\right)=\sum_{l=1}^{L} f_{R}^{l}(r) A c^{l}
$$

where $r=\sqrt{\left(x_{1}^{\prime}-x_{1}^{l}\right)^{2}+\left(x_{2}^{\prime}-x_{2}^{l}\right)^{2}}$ with $x^{\prime} \in \Gamma$ and $x^{l} \in \Omega$. Several RBF have been tried and, in this case, the inverse quadratic function $\left(f_{R}(r)=1 / \sqrt{r^{2}+c^{2}} ; c=\sqrt{2}\right)$ has been found to represent best the behaviour of $w_{3, \alpha}\left(\mathbf{x}^{\prime}\right)$. This is an uncertain method, as extrapolation of functions is not the best for accuracy; but providing that the function is not too irregular and the DRM points are appropriately positioned, it does give good results, as shown in Appendix. It follows that for each geometry it must be performed an accurate convergence study as regards of number of DRM points and their position. The strong points of this method are fast computation time and small memory requirements.

Once the derivatives of the deflection $w_{3, \alpha}$ are computed, both in the domain and at the boundary, it is possible to obtain $N_{\alpha \beta}, \frac{B}{2}\left(w_{3, \alpha} w_{3, \beta}\right)$ and boundary tractions $\left(\dot{t}_{1}, \dot{t}_{2}\right)$.

The derivatives of the quantities $\left(N_{\alpha \beta} w_{3, \beta}\right)$ and $\frac{B}{2}\left(w_{3, \alpha} w_{3, \beta}\right)$ are needed at domain points. Following [17], they are approximated with radial basis function (RBF) as follows:

$$
f\left(w_{3, \alpha}, N_{\alpha \beta}\right)=\sum_{l=1}^{L} f_{R}^{l}(r) A c^{l}
$$

where $f\left(w_{3, \alpha}, N_{\alpha \beta}\right)$ is the generic large deflection term, $f_{R}^{l}(r)$ is the radial basis function; $r=\sqrt{\left(x_{1}-x_{1}^{l}\right)^{2}+\left(x_{2}-x_{2}^{l}\right)^{2}}$ and the $A c^{l}$ are a set of unknown coefficients. The unknown coefficients $A c^{l}$ can be evaluated inverting equation (19) as follows:

$$
\mathbf{A c}=\mathbf{F}_{R}^{-1} f\left(N_{\alpha \beta}, w_{3, \alpha}\right)
$$

where Ac is the matrix of coefficients, $\mathbf{F}_{R}^{-1}$ is the inverse of the matrix of the radial basis functions.

Taking the derivative with respect to the domain point it is possible to write the approximated derivatives as follows: 


$$
f,_{\alpha}\left(w_{3, \alpha}, N_{\alpha \beta}\right)=\mathbf{F}_{R, \alpha} \mathbf{F}_{R}^{-1} f\left(w_{3, \alpha}, N_{\alpha \beta}\right)
$$

where the derivatives $f_{, \alpha}\left(w_{3, \alpha}, N_{\alpha \beta}\right)$ are expressed in terms of $f\left(w_{3, \alpha}, N_{\alpha \beta}\right), \mathbf{F}_{R, \alpha}$ and $\mathbf{F}_{R}^{-1}$ which are known quantities. Several approximating functions have been tested, and the quadratic function $\left(f_{R}(r)=\sqrt{r^{2}+c^{2}} ; c=\right.$ $\sqrt{2}$ ) has been found to be the most accurate.

Once the large deflection terms have been evaluated for step $s$, relaxation technique is introduced as follows:

$$
\dot{L}(s)=\varepsilon \dot{L}(s)-(1-\varepsilon) \dot{L}(s)
$$

where $\varepsilon \in(0,1)$. In this thesis is arbitrarily chosen as $\varepsilon=0.5$.

\section{Domain integrals}

All the domain integrals found are transferred to the boundary by means of the Dual Reciprocity Method as described in [1]. The generic domain integrals can be approximated as follows:

$$
\int_{\Omega} G\left(x^{\prime}, X\right) g(X) d \Omega \simeq \sum_{l=1}^{L} A c^{l} \int_{\Omega} G\left(x^{\prime}, X^{l}\right) \phi(r) d \Omega
$$

where $G$ is the displacement fundamental solution for the domain integral considered (either $U_{\alpha \beta}, W_{i k}, W_{3 k, \alpha}$, $D_{\alpha \beta \gamma}^{m}$ or $\left.D_{i \beta k}^{b}\right) ; g$ is the domain load inclusive of the large deflection term; $A c^{l}$ contains the weighting coefficients and $\phi(r)$ is the approximating function.

Once the function $\phi(r)$ has been chosen, the boundary integral formulation for each pair of points $x^{\prime}, X^{l}$ can be written as follows:

$$
\begin{gathered}
\int_{\Omega} G\left(x^{\prime}, X^{l}\right) \phi\left(x^{\prime}, X^{l}\right) d \Omega= \\
C\left(x^{\prime}\right) \bar{\phi}^{1}\left(x^{\prime}\right) f_{\Gamma} G^{1}\left(x^{\prime}, X^{l}\right) \bar{\phi}^{1}\left(X^{l}\right) d \Gamma-\int_{\Gamma} G^{2}\left(x^{\prime}, X^{l}\right) \bar{\phi}^{2}\left(X^{l}\right) d \Gamma
\end{gathered}
$$

where $\bar{\phi}^{1}, \bar{\phi}^{2}$ are particular solutions for the chosen approximating function, and $G^{1}\left(x^{\prime}, X^{l}\right), G^{2}\left(x^{\prime}, X^{l}\right)$ are the fundamental solution of the original problem.

\section{System matrix}

After performing all the collocations (at boundary and DRM points) the system of equation can be written for each plate as follows:

$$
\begin{gathered}
{\left[\begin{array}{ll}
\mathbf{H}_{5 N \times 5 N}^{b n} & \mathbf{0}_{5 N \times 5 L} \\
\mathbf{H}_{5 L \times 5 N}^{d r m} & \mathbf{I}_{5 L \times 5 L}
\end{array}\right]\left\{\begin{array}{l}
\mathbf{d}_{5 N}^{b n} \\
\mathbf{d}_{5 L}^{d r m}
\end{array}\right\}-\left[\begin{array}{ll}
\mathbf{G}_{5 N \times 15 N_{e}}^{b n} & \mathbf{0}_{5 N \times 5 L} \\
\mathbf{G}_{5 L \times 15 N_{e}}^{d r m} & \mathbf{0}_{5 L \times 5 L}^{d r m}
\end{array}\right]\left\{\begin{array}{l}
\mathbf{b}_{15 N_{e}}^{b n} \\
\mathbf{0}_{5 L}
\end{array}\right\}=} \\
-\left[\begin{array}{ll}
\mathbf{G}_{5 N \times 15 N_{e}}^{b n} & \mathbf{0}_{5 N \times 5 L} \\
\mathbf{G}_{5 L \times 15 N_{e}}^{d r m} & \mathbf{0}_{5 L \times 5 L}^{d r m}
\end{array}\right]\left\{\begin{array}{l}
\dot{\mathbf{b}}_{15 N_{e}}^{b n} \\
\mathbf{0}_{5 L}^{d r}
\end{array}\right\}+\mathbf{F}_{5(N+L) \times 5 L}\left(\mathbf{L}_{5 L}+\dot{\mathbf{L}}_{5 L}\right)
\end{gathered}
$$

where $N$ is the number of boundary source points; $L$ is the number of DRM points; $\mathbf{H}^{b n}$, $\mathbf{G}^{b n}$, and $\mathbf{H}^{d r m}$, $\mathbf{G}^{d r m}$ are the boundary element influence matrix when collocating on the boundary (including the crack) and in the domain respectively; $\mathbf{I}$ is the identity matrix; $\mathbf{d}^{b n}$ and $\mathbf{d}^{d r m}$ are displacement vectors for boundary and DRM points; $\mathbf{b}^{b n}$ is the traction vector for boundary points; $\dot{\mathbf{b}}^{b n}$ contains the in-plane boundary tractions $\dot{t}$; the matrix $\mathbf{F}$ is the influence matrix of the domain loads calculated with the DRM; $\mathbf{L}$ contains the prescribed domain loads and $\dot{\mathbf{L}}$ the large deflection domain terms.

Building the system for each plate, applying the boundary conditions and adding the appropriate equilibrium and compatibility equations at join lines, it is possible to write, for a generic step $s$ : 


\begin{tabular}{|c|c|c|c|c|}
\hline.. & 0 & 0 & 0 & 0 \\
\hline 0 & $\mathbf{A}^{j}$ & 0 & 0 & 0 \\
\hline 0 & 0 &.. & 0 & 0 \\
\hline 0 & 0 & 0 & $\mathbf{A}^{k}$ & 0 \\
\hline 0 & 0 & 0 & 0 &.. \\
\hline 0 & $\mathbf{J}^{j k}$ & 0 & $\mathbf{J}^{j k}$ & 0 \\
\hline
\end{tabular}$=$\begin{tabular}{|c|}
\hline $\mathbf{F}^{j}\left(\mathbf{L}_{(s)}^{j}+\dot{\mathbf{L}}_{(s-1)}^{j}\right)$ \\
..$\cdot$ \\
\hline $\mathbf{F}^{k}\left(\mathbf{L}_{(s)}^{k}+\dot{\mathbf{L}}_{(s-1)}^{k}\right)$ \\
\hline.$\cdot$ \\
\hline 0 \\
\hline $\mathbf{b}_{(s)}^{j}-\dot{\mathbf{b}}_{(s-1)}^{j}$ \\
.. \\
\hline $\mathbf{b}_{(s)}^{k}-\dot{\mathbf{b}}_{(s-1)}^{k}$ \\
\hline.. \\
\hline 0 \\
\hline
\end{tabular}

where $\mathbf{A}^{j}, \mathbf{A}^{k}$ are the matrix containing coefficients for the unknowns; $\mathbf{F}^{j}, \mathbf{F}^{k}$ are matrix obtained by DRM technique for the domain loads; $\mathbf{L}_{(s)}^{j}, \mathbf{L}_{(s)}^{k}$ contain the prescribed domain loads at step $s ; \dot{\mathbf{L}}_{(s-1)}^{j} \dot{\mathbf{L}}_{(s-1)}^{k}$ contain the large deflection domain contribution evaluated at step $s-1 ; \mathbf{b}_{(s)}^{j}, \mathbf{b}_{(s)}^{k}$ are the known terms due to boundary conditions at step $s ; \dot{\mathbf{b}}_{(s-1)}^{j}, \dot{\mathbf{b}}_{(s-1)}^{k}$ are the terms due to large deflection boundary traction evaluated at step $s-1$, and finally $\mathbf{J}^{j k}$ are the matrix containing the joining conditions between plates $j$ and $k$.

\section{Evaluation of fracture parameters}

Once the system (25) has been solved and the solution at the boundary is obtained, the fracture parameters can be evaluated. In large deflection analysis, higher order terms have to be considered in the equations describing the stress and displacement field at the crack tip, as the membrane stress resultants depend on derivatives of deflection too. The SIFs cannot be defined purely in terms of of the crack face displacements as in the linear elastic case. Huy [5] proved that in Kirchhoff plate theory the stress singularity can be described by standard SIFs, but this is not true in shear deformable plate theory. Dirgantara and Aliabadi [12] presented the J-integral for linear elastic shear deformable plates. Here, we present the $J$-integral for large deformation theory, where the energy associated to the large deflection can been included as follows:

$$
\begin{aligned}
J^{m}=\int_{\Gamma}\left[N_{\alpha \beta} \frac{1}{2}\left(u_{\alpha, \beta}+u_{\beta, \alpha}\right) n_{n}+N_{\alpha \beta} \frac{1}{2}\left(w_{3, \alpha} w_{3, \beta}\right) n_{n}+\right. \\
\left.+t_{\beta} u_{\beta, r}\right] d \Gamma-\int_{\Omega} f_{\beta} u_{\beta, n} d \Omega \\
J^{b}=\int_{\Gamma}\left[\left(Q_{\alpha}\left(w_{\alpha}+w_{3, \alpha}\right)+M_{\alpha \beta} \frac{1}{2}\left(w_{\alpha, \beta}+w_{\beta, \alpha}\right)\right) n_{n}+\right. \\
\left.-p_{k} w_{k, n} d \Gamma\right]-\int_{\Omega}\left(q_{k}+\delta_{3 k}\left(N_{\alpha \beta} w_{3, \beta}\right), \alpha\right. \\
\end{aligned}
$$

where $J^{m}$ and $J^{b}$ stand for membrane and bending $J$-integral respectively. The integration path is taken as a circle centered in the crack tip as shown in figure (4). The domain integrations are performed in the region contained by the path as shown in the same figure.

For the domain integral in equation (26), the integration is performed using standard integration techniques. The domain integral $\int_{\Omega}\left(N_{\alpha \beta} w_{3, \beta}\right)_{, \alpha} w_{3, n} d \Omega$ in equation (27), is singular at the crack-tip. By transforming $d \Omega$ in polar coordinates, the order of singularity is reduced and the integral can be computed.

By means of the separation technique proposed by Rigby and Aliabadi [3] for 3D, and by Dirgantara and Aliabadi for plates [12] it is possible to evaluate independently the components of the $J$-integral. Although in [3] and [12] the procedure is shown for small deflection only, it is possible to follow a similar procedure for large deflection by decomposing the total stresses and strains.

The $J$-integral for membrane can be then written as sum of two parts as follows:

$$
J^{m}=J_{1}^{m}+J_{2}^{m}
$$

where $J_{1}^{m}$ correspond to mode I and $J_{2}^{m}$ correspond to mode II. 


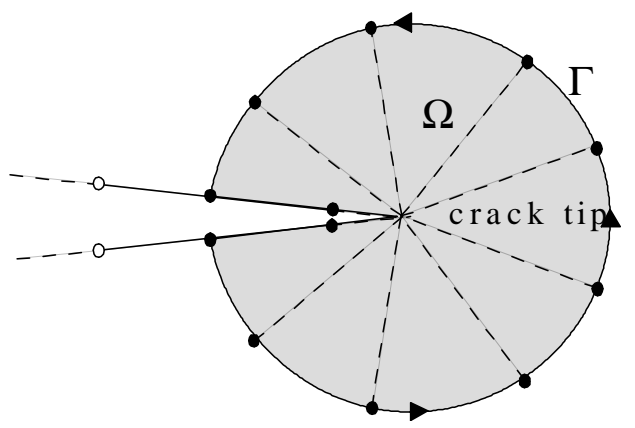

Figure 4: J-integral boundary and domain discretization

In order to compare with classical stress resultant intensity factors the following quantities can be defined:

$$
\begin{aligned}
\left(K_{1}^{m}\right)^{*} & =\sqrt{\frac{J_{1}^{m} E}{1-\nu^{2}}} \\
\left(K_{2}^{m}\right)^{*} & =\sqrt{\frac{J_{2}^{m} E}{1-\nu^{2}}}
\end{aligned}
$$

where $\left(K_{1}^{m}\right)^{*}$ and $\left(K_{2}^{m}\right)^{*}$ indicate the dissipation of energy at crack tip rather than the stress singularity. The above quantities may be used to perform fatigue analyses and crack growth simulations, similarly to standard SIFs. Although it is not strictly correct, it will give an idea of the large deflection effects on the crack. 


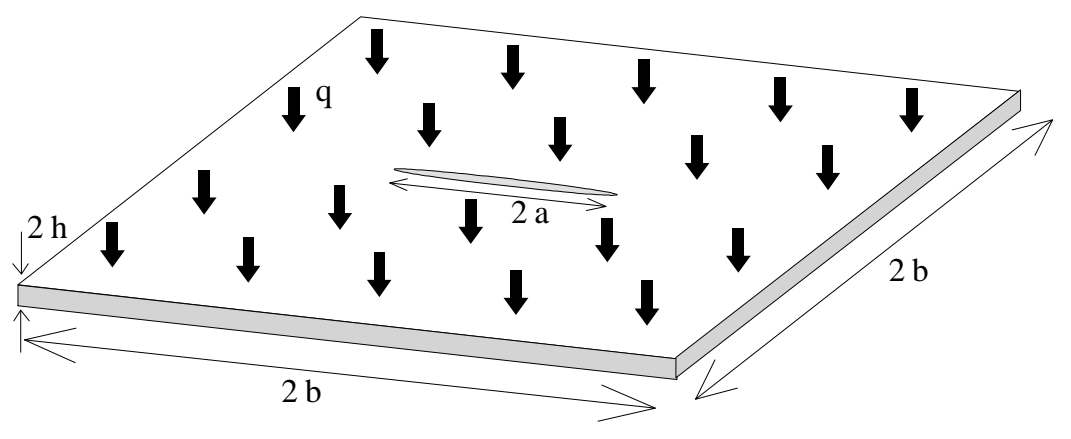

Figure 5: Simply supported plate under transverse shear load

\section{Large deflection analysis of a simply supported square plate with a central crack under transverse uniform pressure}

Consider the simply supported square plate presented in figure ( 5 which properties are $E / q=10,000$, Poisson's ratio $\nu=0.3$, and $h / b=1 / 6$. The central crack is $2 a$ long, where $a / b=0.2 ; 0.4 ; 0.6$ and 0.8 . First the case with $a / b=0.2$ is considered. Four different models are employed:

1. 16 elements on external boundary, 8 elements on each crack surface (total 64 boundary points), 16 DRM points;

2. 16 elements on external boundary, 8 elements on each crack surface (total 64 boundary points), 36 DRM points;

3. 32 elements on external boundary, 16 elements on each crack surface (total 128 boundary points), 36 DRM points;

4. 32 elements on external boundary, 16 elements on each crack surface (total 128 boundary points), 64 DRM points;

The influence of the load increment has been studied for all above models. In all cases convergence has been achieved for load step $=80$. Consider the case $E=1,000 M P a, q=0.1 M p a$ and $b=1 \mathrm{~mm}$ For model 4 , in figure (6), the radial component of the normal stress resultant in crack tip polar coordinate $\left(N_{r r}(r, \theta)\right)$ at different angles $\left(\theta=0^{\circ}, 45^{\circ}, 90^{\circ}\right)$, is shown against the distance $r$, for 10, 40 and 80 load steps.

In figure (7), $N_{r r}\left(r, 0^{\circ}\right)$ and $N_{\theta \theta}\left(r, 0^{\circ}\right)$ are shown for all models. It can be seen how little is the difference between model 2 and model 3; accurate results can be found for the stress field already with 64 boundary points, while the dependency from the DRM points is stronger.

In figure (8), $N_{r r}\left(r, 0^{\circ}\right)$ is shown for $a / b=0.2,0.4$ and 0.6 , where model 4 has been used for all cases.

Several integration path have been tried and convergence has been found for (path-radius) $/ a=1 / 16$. In figure (9) $\left(K_{1}^{m}\right)^{*}$ and $K_{1}^{b}$ for $a / b=0.2$ are plotted for each model. It can be seen how the bending is similar for all models, while the membrane behaviour is more sensitive to large deflection approximations.

In table (1) results are presented for various crack sizes, where model 4 was used in all cases. It can be seen how the large deflection effects increase with crack size, the plate is weaker thus it bends more. It can be noticed how $K_{1}^{b}$ becomes lower than linear cases with the increase of the crack size (and consequently of large deflection effects). For $a / b=0.6,0.8$ the values of $w_{3}^{\max }$ are similar, but the value of $\left(K_{1}^{m}\right)^{*}$ for $a / b=0.8$ is almost double than for $a / b=0.6$. The contribution of domain integral $\int_{\Omega}\left(N_{\alpha \beta} w_{3, \beta}\right)_{, \alpha} w_{3, n} d \Omega$ to $J^{b}$ has been found negligible for the cases examined.

In figure $(10),\left(K_{1}^{m}\right)^{*}$ for $a / b=0.4$ is plotted against the load steps. 

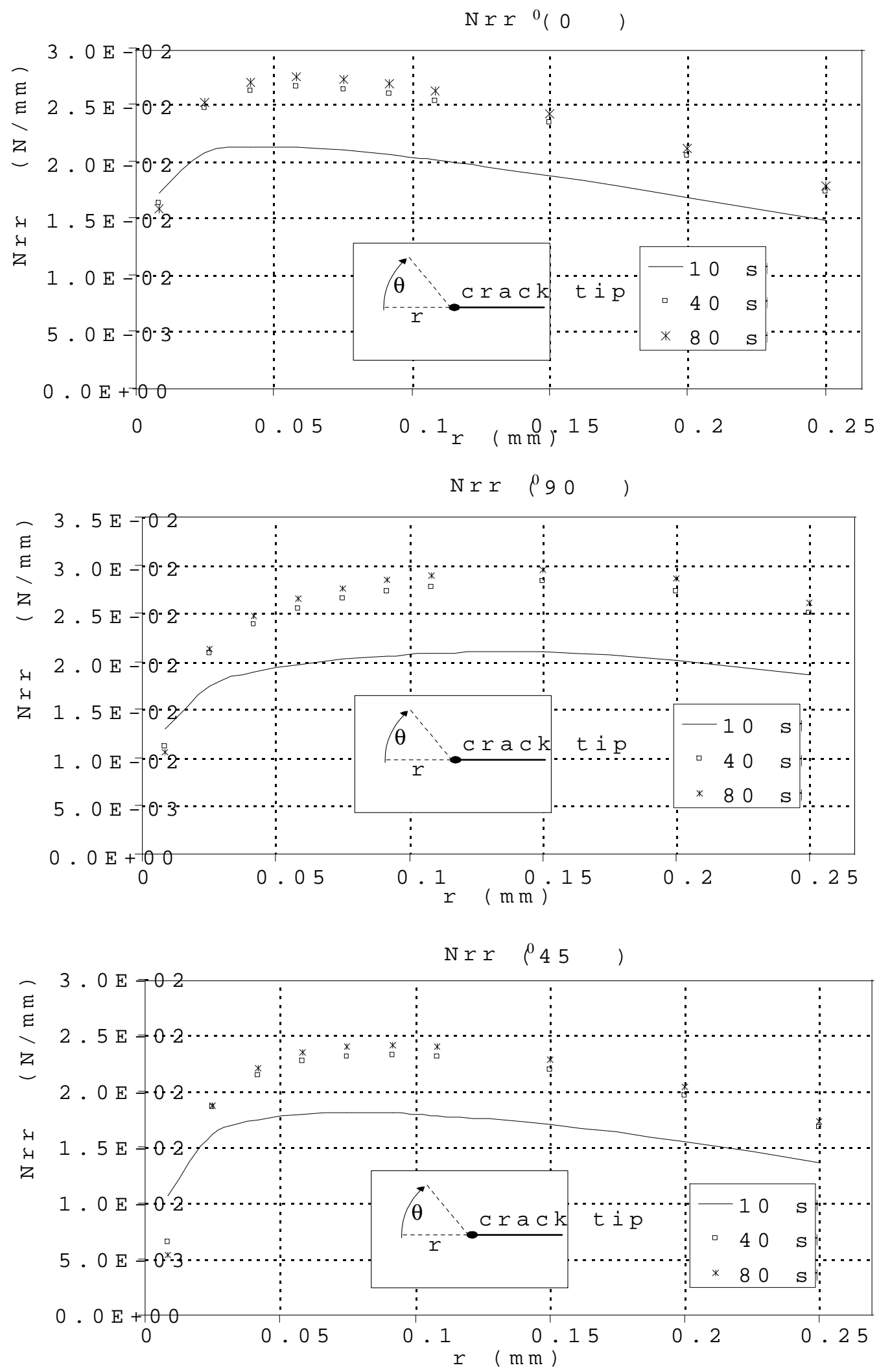

Figure 6: Simply supported plate, model 4: Normal stress resultant $N_{r r}(r, \theta)$ at crack tip 


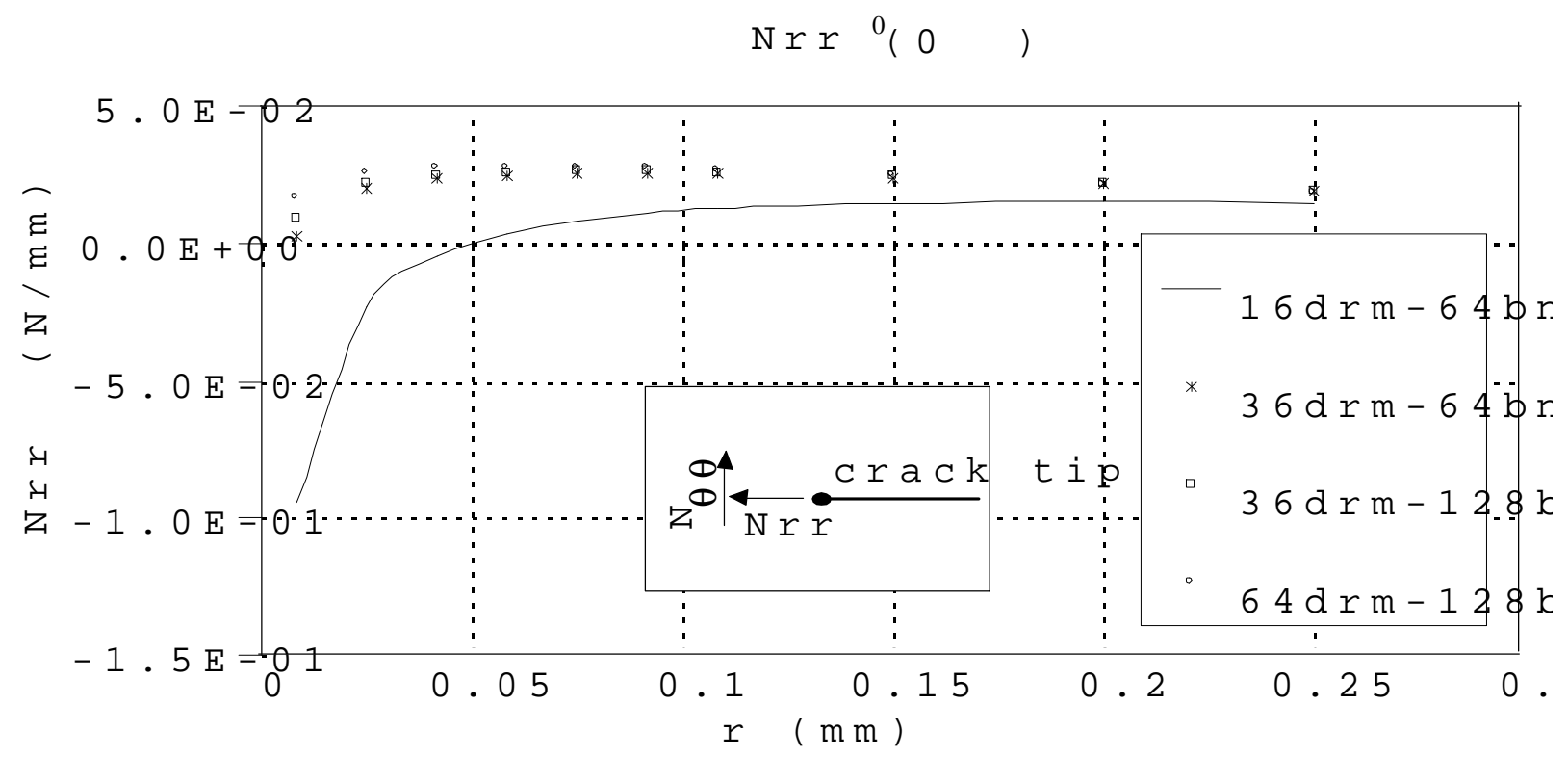

${ }^{N} \theta \theta \quad{ }^{0}(0)$

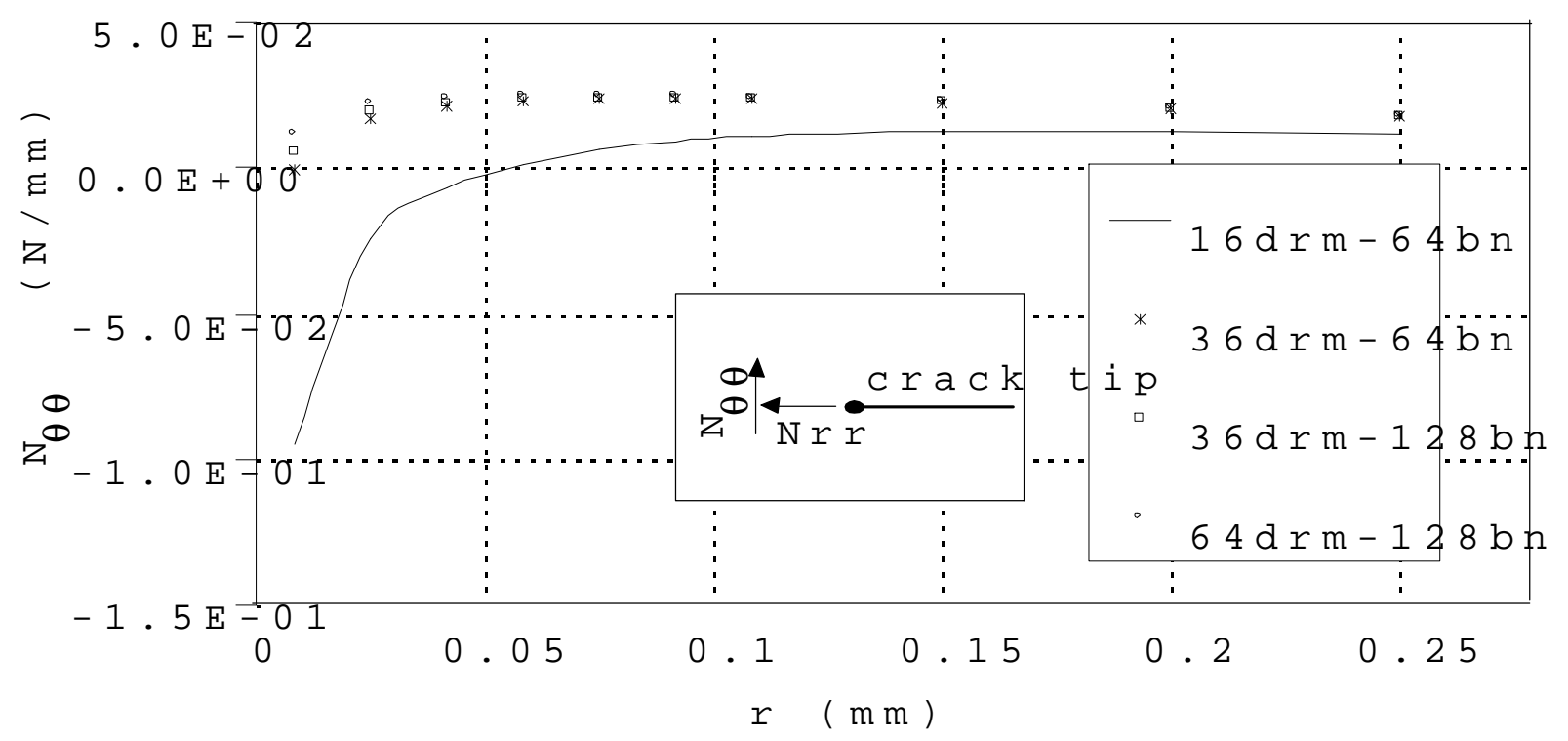

Figure 7: Simply supported plate: normal stress resultants near crack tip for $\theta=0^{\circ}$ 


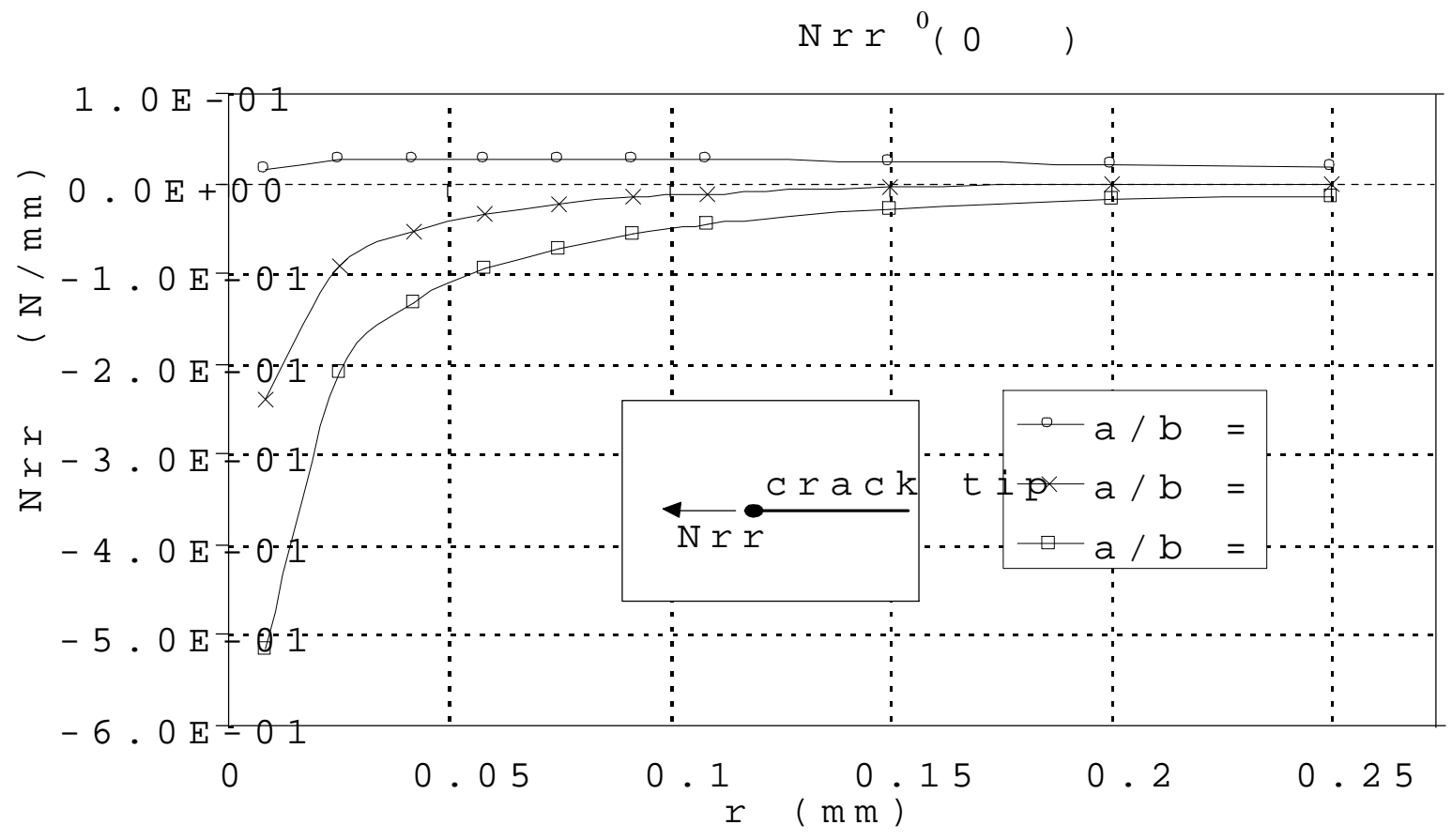

Figure 8: Simply supported plate: $N_{r r}\left(r, 0^{\circ}\right)$ for various $a / b$

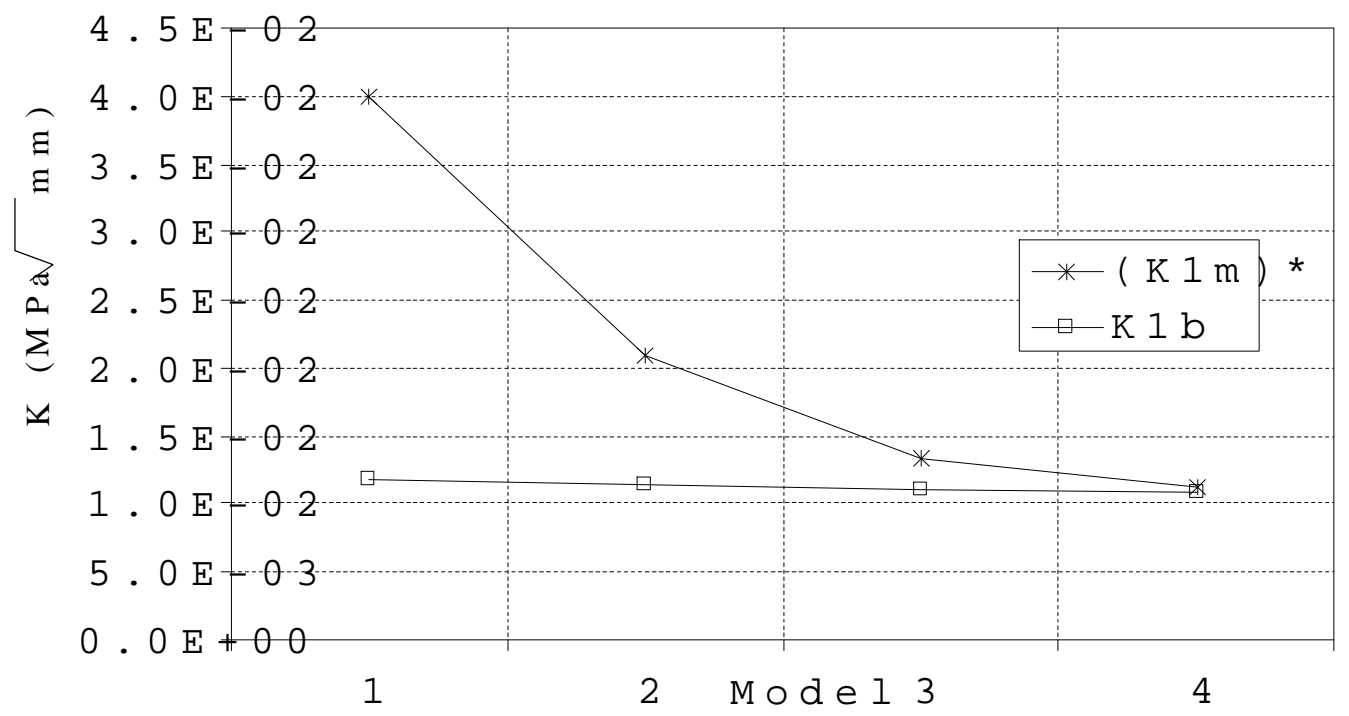

Figure 9: Simply supported plate $(a / b=0.2):\left(K_{1}^{m}\right)^{*}$ and $K_{1}^{b}$ for different models 


\begin{tabular}{|c|c|c|c|c|}
\hline$a / b$ & $\left(K_{1}^{m}\right)^{*}$ & $K_{1}^{b}$ & $K_{1}^{b}$ linear $[13]$ & $w_{3}^{\max }$ \\
\hline 0.2 & $1.13 E-02$ & $1.09 E-02$ & $1.09 E-02$ & $1.85 E-02$ \\
\hline 0.4 & $1.34 E-02$ & $8.25 E-03$ & $1.30 E-02$ & $1.90 E-02$ \\
\hline 0.6 & $1.85 E-02$ & $9.33 E-03$ & $1.29 E-02$ & $2.16 E-02$ \\
\hline 0.8 & $3.58 E-02$ & $6.40 E-03$ & $9.83 E-03$ & $2.17 E-02$ \\
\hline
\end{tabular}

Table 1: Simply supported square plate: results for various a/b comparisons

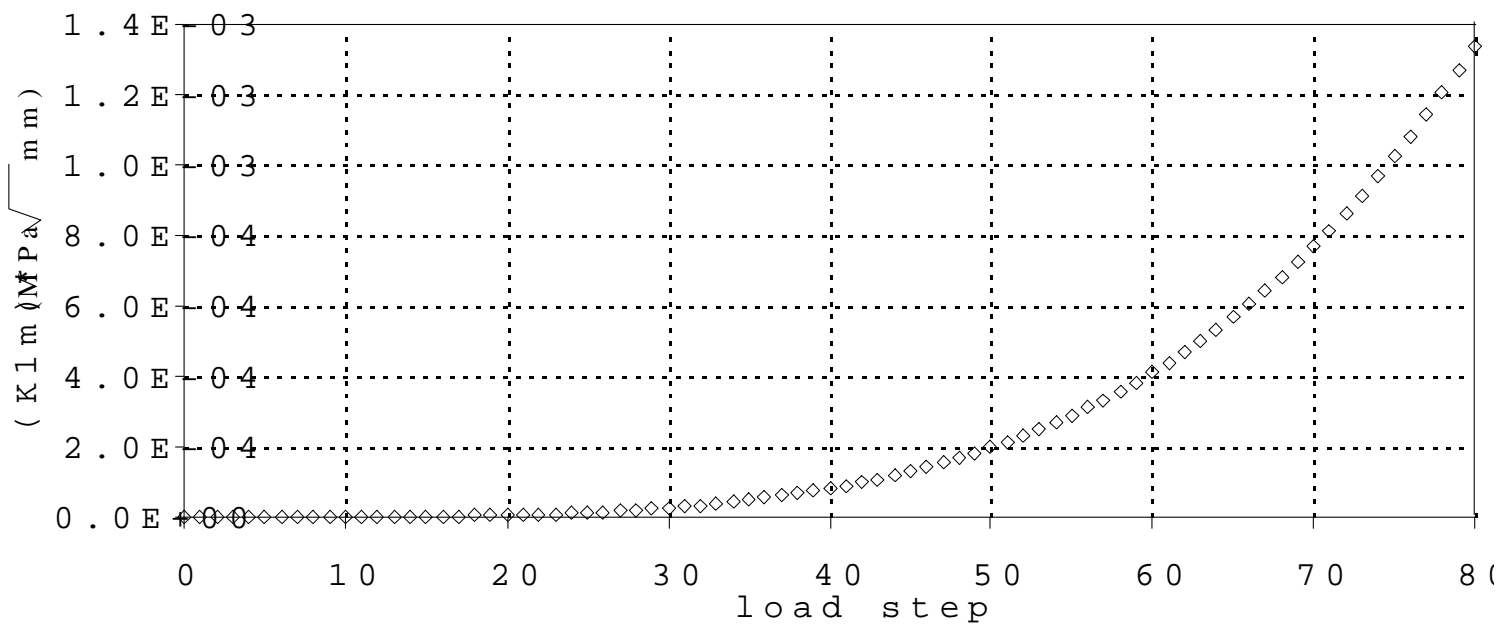

Figure 10: Simply supported plate: $\left(K_{1}^{m}\right)^{*}$ for $a / b=0.4$ against load steps

\section{Comments on the use of DRM points}

Several different distributions of DRM points were studies here. It was found that when the total number of DRM points is close or equal to the total number of boundary points the resulting solutions are stable. However, so cases where the DRM point are much larger (about twice or more) than the boundary point the solutions are unstable. This instability could be in part due to the integration of near singularity which arise from the proximity of internal points to the boundary. Normally, for the best solutions using numerical integration it is advisable for the distance between the internal points and the boundary to be half of the size of the element on the boundary. Large number of DRM internal points will result in large portion of the final system matrix being devoted to the modelling of the domain integral than boundary values. Hence small inaccuracies in the DRM approximation will affect the solution in a disproportionate manner.

Nevertheless, the solution is found to be good when the number of DRM points does not exceed the number of boundary source points. 


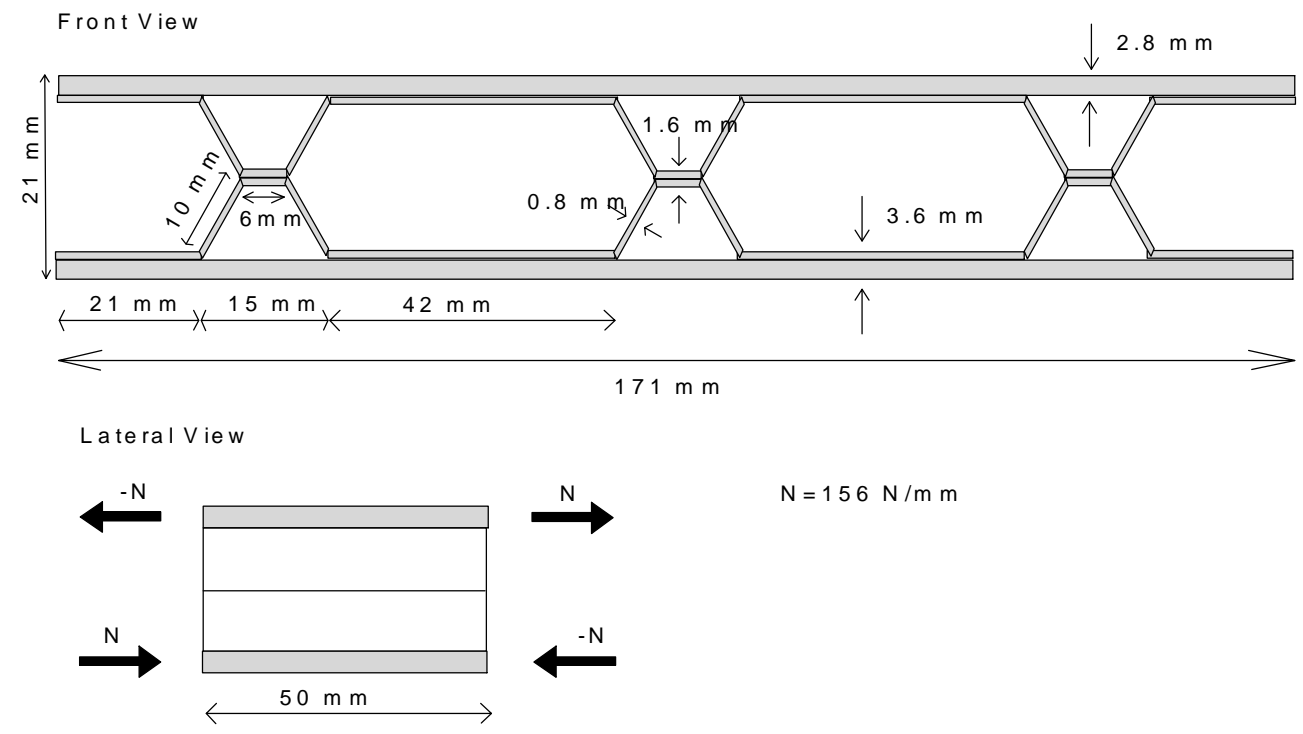

Figure 11: X-core section: geometry and load

\section{Large deflection analysis of X-core structure with central crack under bending load}

The structure presented in figure (11) is modelled with 29 flat plates of titanium alloy (Young's modulus $E=110 \mathrm{GPa}$; Poisson's ratio $v=0.3$ ) of different thicknesses assembled into a structure which is $w=171 \mathrm{~mm}$ wide $(x)$ by $d=50 \mathrm{~mm}$ deep $(y)$ and $h=17.9 \mathrm{~mm}$ high $(z)$. It is subject to a bending moment of $477 \mathrm{kN} \times \mathrm{mm}$, generated by applying a uniform compression load $(N=156 \mathrm{~N} / \mathrm{mm})$ along the edges of the lower skin and uniform tension load of same intensity along the edges of the upper skin. Each plate has been modelled with 16 quadratic boundary elements and 16 DRM points. A central embedded crack $2 a=5 \mathrm{~mm}$ placed as shown in figure (12). 36 DRM points have been added to the cracked plate. Convergence on load increment was obtained using 100 steps. The results are shown in figure (13), where the parameter $\left(K_{1}^{m}\right)^{*}$ is normalized with $N / h \sqrt{\pi a}$ and plotted against the load. The normalized membrane stress resultant intensity factors $\left(K_{1}^{m}\right)$ is presented as well for comparisons. Although a direct comparison between these two parameters is not possible a priori, they give an idea of how the energy related to the large deflection case is significantly higher. 


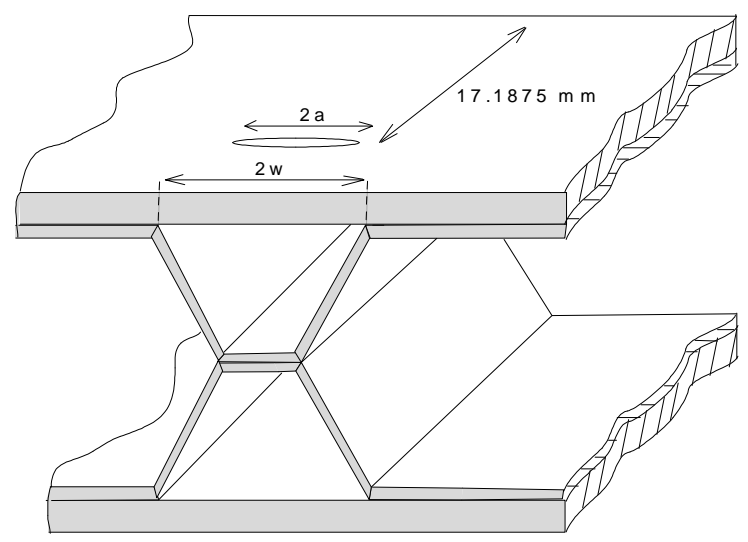

Figure 12: X-core section: crack geometry

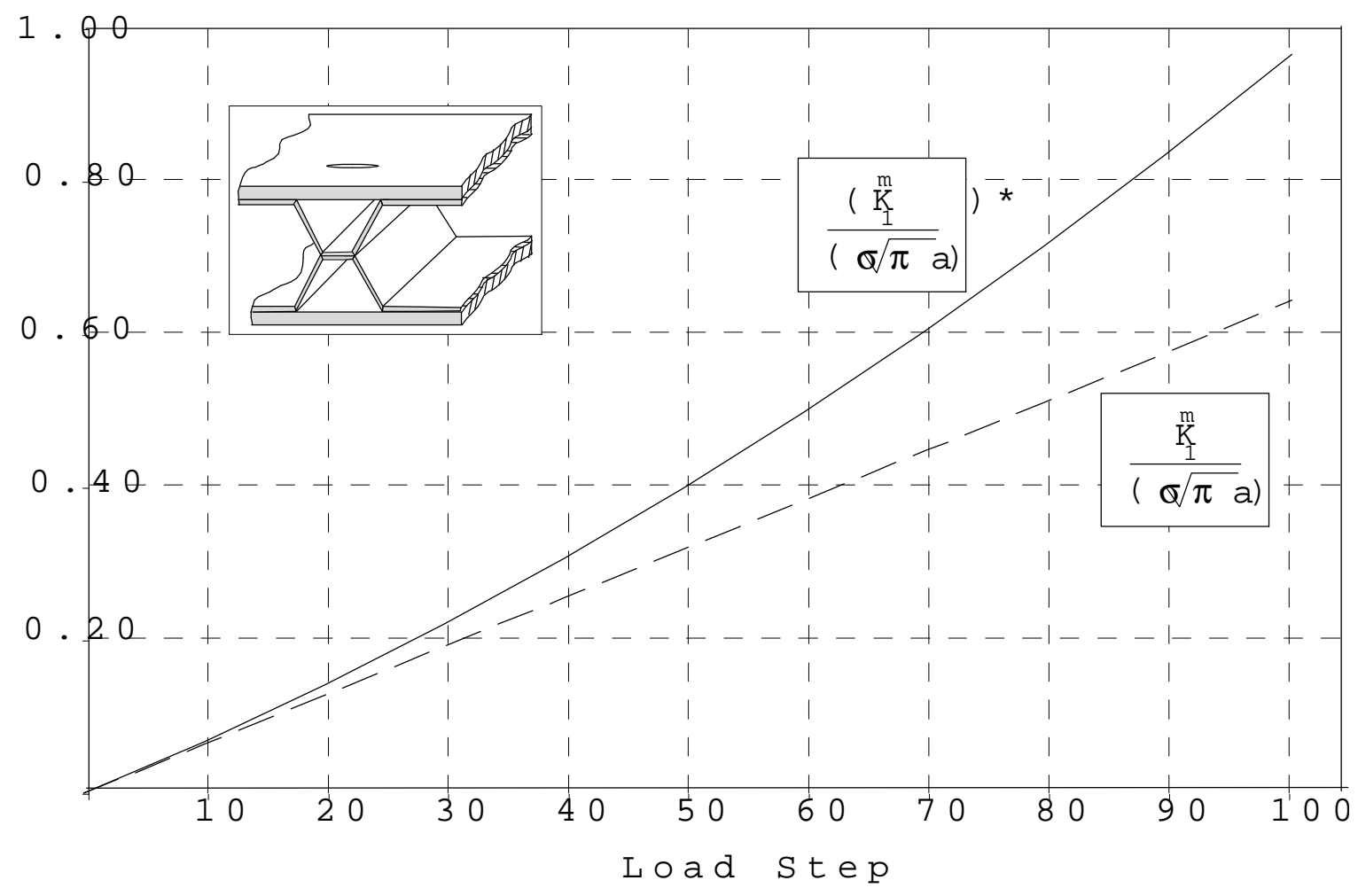

Figure 13: X-core with central cral under bending load: large deflection stress intensity factors 


\section{Conclusions}

The Dual Boundary Element Method was combined with a multi-region formulation to simulate plate assembly undergoing large deflection. The system of equation was linearized using an incremental load approach and Radial Basis Functions were used to approximate the derivatives of the large deflection terms. The Dual Reciprocity Method was successfully used to transfer to the boundary the large deflection domain integrals. The stress field at the crack tip in large deflection has not been yet solved; a rigorous definition of SIFs was not found possible, as higher order terms need to be included in the equations describing the crack tip stress field. Therefore, once the solution at the boundary was obtained, fracture parameters were extracted using a J-integral formulation for shear deformable plates in large deflection.

\section{Appendix A}

\section{Plate bending fundamental solutions for displacement integral equations}

The expressions for the kernels $W_{i j}$ and $P_{i j}$ are given by Vander Weeën [15] and can be written as follows:

$$
\begin{gathered}
W_{\alpha \beta}=\frac{1}{8 \pi D(1-\nu)}\left\{[8 B(z)-(1-\nu)(2 \ln z-1)] \delta_{\alpha \beta}+\right. \\
\left.-[8 A(z)+2(1-\nu)] r_{, \alpha} r_{, \beta}\right\} \\
W_{\alpha 3}=-W_{3 \alpha}=\frac{1}{8 \pi D}(2 \ln z-1) r r_{, \alpha} \\
W_{33}=\frac{1}{8 \pi D(1-\nu) \lambda^{2}}\left[(1-\nu) z^{2}(\ln z-1)-8 \ln z\right]
\end{gathered}
$$

and

$$
\begin{gathered}
P_{\gamma \alpha}=\frac{-1}{4 \pi r}\left[\left(4 A(z)+2 z K_{1}(z)+1-\nu\right)\left(\delta_{\alpha \gamma} r_{, n}+r_{, \alpha} n_{\gamma}\right)+\right. \\
\left.+(4 A(z)+1+\nu) r_{, \gamma} n_{\alpha}-2\left(8 A(z)+2 z K_{1}(z)+1-\nu\right) r_{, \alpha} r_{, \gamma} r_{, n}\right] \\
P_{\gamma \beta}=\frac{\lambda^{2}}{2 \pi}\left[B(z) n_{\gamma}-A(z) r_{, \gamma} r_{, n}\right] \\
P_{3 \alpha}=\frac{-(1-\nu)}{8 \pi}\left[\left(2 \frac{(1+\nu)}{(1-\nu)} \ln z-1\right) n_{\alpha}+2 r_{, \alpha} r_{, n}\right] \\
P_{33}=\frac{-1}{2 \pi r} r_{, n}
\end{gathered}
$$

The expression for the kernels derivatives $W_{3 j, \theta}$ and $P_{3 j, \theta}$ are written as

$$
\begin{gathered}
W_{3 \alpha, \theta}=-\frac{1}{8 \pi D}\left(2 r_{, \theta} r_{, \alpha}+(2 \ln z-1) \delta_{\alpha \theta}\right) \\
W_{33, \theta}=\frac{1}{8 \pi D \lambda} r_{, \theta}\left[(2 \ln z-1)-\frac{8}{z(1-\nu)}\right] \\
P_{3 \alpha, \theta}=\frac{-(1-\nu)}{4 \pi r}\left[\left(\frac{(1+\nu) r_{, \theta}}{(1-\nu)}\right) n_{\alpha}+r_{, \alpha} n_{\theta}-3 r_{, \theta} r_{, n} r_{, \alpha}+\delta_{\alpha \theta} r_{, n}\right] \\
P_{33, \theta}=\frac{-1}{2 \pi r^{2}}\left[n_{\theta}-2 r_{, \theta} r_{, n}\right]
\end{gathered}
$$




\section{Plate bending fundamental solutions for traction integral equations}

The fundamental solutions for the traction integral equations are linear combination of the derivatives of the fundamental solution for displacement integral equations; the expression of kernels $D_{i j k}^{b}, S_{i j k}^{b}$ are :

$$
\begin{gathered}
D_{\alpha \beta \gamma}^{b}=\frac{1}{4 \pi r}\left[\left(4 A(z)+2 z K_{1}(z)+1-\nu\right)\left(\delta_{\beta \gamma} r_{, \alpha}+\delta_{\alpha \gamma} r_{, \beta}\right)+\right. \\
\left.-2\left(8 A(z)+2 z K_{1}(z)+1-\nu\right) r_{, \alpha} r_{, \beta} r_{, \gamma}+(4 A(z)+1+\nu) \delta_{\alpha \beta} r_{, \gamma}\right] \\
D_{\alpha \beta 3}^{b}=\frac{-(1-\nu)}{8 \pi}\left[\left(2 \frac{(1+\nu)}{(1-\nu)} \ln z-1\right) \delta_{\alpha \beta}+2 r_{, \alpha} r_{, \beta}\right] \\
D_{3 \beta \gamma}^{b}=\frac{\lambda^{2}}{2 \pi}\left[B(z) \delta_{\gamma \beta}-A(z) r_{, \gamma} r_{, \beta}\right] \\
D_{3 \beta 3}^{b}=\frac{1}{2 \pi r} r_{, \beta} \\
+(4 A(z)+1+3 \nu) \delta_{\alpha \beta} n_{\gamma}-\left(16 A(z)+6 z K_{1}(z)+z^{2} K_{0}(z)+2-2 \nu\right) \times \\
\times\left[\left(n_{\alpha} r_{, \beta}+n_{\beta} r_{, \alpha}\right) r_{, \gamma}+\left(\delta_{\gamma \alpha} r_{, \beta}+\delta_{\gamma \beta} r_{, \alpha}\right) r_{, n}\right]+ \\
-2\left(8 A(z)+2 z K_{1}(z)+1+\nu\right)\left(\delta_{\alpha \beta} r_{, \gamma} r_{, n}+n_{\gamma} r_{, \alpha} r_{, \beta}\right)+ \\
\left.+4\left(24 A(z)+8 z K_{1}(z)+z^{2} K_{0}(z)+2-2 \nu\right) r_{, \alpha} r_{, \beta} r_{, \gamma} r_{, n}\right\} \\
S_{\alpha \beta \gamma}^{b}=\frac{D(1-\nu)}{4 \pi r^{2}}\left\{\left(4 A(z)+2 z K_{1}(z)+1-\nu\right)\left(\delta_{\gamma \alpha} n_{\beta}+\delta_{\gamma \beta} n_{\alpha}\right)+\right. \\
\left.-2\left(4 A(z)+z K_{1}(z)\right) r_{, \alpha} r_{, \beta} r_{, n}+2 A(z) \delta_{\alpha \beta} r_{, n}\right] \\
S_{3 \beta \gamma}^{b}=\frac{-D(1-\nu) \lambda^{2}}{4 \pi r}\left[\left(2 A(z)+z K_{1}(z)\right)\left(\delta_{\gamma \beta} r_{, n}+r_{, \gamma} n_{\beta}\right)+\right. \\
\left.+2 A(z) n_{\gamma} r_{, \beta}-2\left(4 A(z)+z K_{1}(z)\right) r_{, \gamma} r_{, \beta} r_{, n}\right] \\
S_{3 \beta 3}^{b}=\frac{D(1-\nu) \lambda^{2}}{4 \pi r^{2}}\left[\left(z^{2} B(z)+1\right) n_{\beta}-\left(z^{2} A(z)+2\right) r_{, \beta} r_{, n}\right]
\end{gathered}
$$

\section{Bessel functions}

The functions $A(z)$ and $B(z)$ can be written in term of the Bessel functions and are defined as follows:

$$
\begin{aligned}
& A(z)=K_{0}(z)+\frac{2}{z}\left[K_{1}(z)-\frac{1}{z}\right] \\
& B(z)=K_{0}(z)+\frac{1}{z}\left[K_{1}(z)-\frac{1}{z}\right]
\end{aligned}
$$

in which $K_{0}(z)$ and $K_{1}(z)$ are modified Bessel functions of the second kind, $z=\lambda r, \lambda=\sqrt{10} / h, r$ is the absolute distance between the source and the field points, $r_{, \alpha}=r_{\alpha} / r$, where $r_{\alpha}=x_{\alpha}(\mathbf{x})-x_{\alpha}\left(\mathbf{x}^{\prime}\right)$ and $r_{, n}=r_{, \alpha} n_{\alpha}$. 
Expanding the modified Bessel functions for small arguments:

$$
\begin{gathered}
K_{0}(z)=\left[-\gamma-\ln \left(\frac{z}{2}\right)\right]+\left[-\gamma+1-\ln \left(\frac{z}{2}\right)\right] \frac{\left(z^{2} / 4\right)}{(1 !)^{2}}+ \\
+\left[-\gamma+1+\frac{1}{2}-\ln \left(\frac{z}{2}\right)\right] \frac{\left(z^{2} / 4\right)^{2}}{(2 !)^{2}}+ \\
+\left[-\gamma+1+\frac{1}{2}+\frac{1}{3}-\ln \left(\frac{z}{2}\right)\right] \frac{\left(z^{2} / 4\right)^{3}}{(3 !)^{2}}+\cdots \\
K_{1}(z)=\frac{1}{z}-\left[-\gamma+\frac{1}{2}-\ln \left(\frac{z}{2}\right)\right] \frac{\left(z^{2} / 4\right)^{1 / 2}}{0 ! 1 !}+ \\
-\left[-\gamma+1+\frac{1}{4}-\ln \left(\frac{z}{2}\right)\right] \frac{\left(z^{2} / 4\right)^{3 / 2}}{1 ! 2 !}+ \\
-\left[-\gamma+1+\frac{1}{2}+\frac{1}{6}-\ln \left(\frac{z}{2}\right)\right] \frac{\left(z^{2} / 4\right)^{5 / 2}}{2 ! 3 !}+\cdots
\end{gathered}
$$

where $\gamma=0.5772156649$ is the Euler constant. Substituting in the kernels and taking the limit as $r \rightarrow 0$ :

$$
\begin{gathered}
\lim _{r \rightarrow 0} A(z)=\frac{-1}{2} \\
\lim _{r \rightarrow 0} B(z)=-\frac{1}{2}\left[\lim _{r \rightarrow 0} \ln \left(\frac{z}{2}\right)+\gamma+\frac{1}{2}\right]
\end{gathered}
$$

As it can be seen, $A(z)$ is a smooth function, whereas, $B(z)$ is a weakly singular $O(\ln r)$. Therefore kernels $W_{i j}, D_{3 \beta \gamma}^{b}, D_{3 \beta \gamma}^{b}$ are weakly singular $O(\ln r)$; kernels $P_{i j}, D_{\alpha \beta \gamma}^{b}, D_{3 \beta 3}^{b}, S_{\alpha \beta 3}^{b}, S_{3 \beta \gamma}^{b}$, have a strong (Cauchy principal value) singularity $O(1 / r)$; while kernels $S_{\alpha \beta \gamma}^{b}, S_{3 \beta 3}^{b}$ are hyper-singular of the order $O\left(1 / r^{2}+\ln r\right)$. The modified Bessel functions are evaluated using polynomial approximations given by Abramowitz and Stegun.

\section{Membrane fundamental solutions for displacement integral equations}

The expressions for the kernels $U_{\theta \alpha}$ and $T_{\theta \alpha}$ are well known (Kelvin solution) for two-dimensional plane stress problems, and are given as:

$$
\begin{gathered}
U_{\theta \alpha}=\frac{1}{4 \pi B(1-\nu)}\left[(3-\nu) \ln \left(\frac{1}{r}\right) \delta_{\theta \alpha}+(1+\nu) r_{, \theta} r_{, \alpha}\right] \\
T_{\theta \alpha}=-\frac{1}{4 \pi r}\left\{r_{, n}\left[(1-\nu) \delta_{\theta \alpha}+2(1+\nu) r_{, \theta} r_{, \alpha}\right]+\right. \\
\left.+(1-\nu)\left[n_{\theta} r_{, \alpha}-n_{\alpha} r_{, \theta}\right]\right\}
\end{gathered}
$$

where $U_{\theta \alpha}$ are weakly singular kernels of order $O\left(\ln \frac{1}{r}\right)$ and $T_{\theta \alpha}$ are strongly singular in order $O(1 / r)$.

\section{Membrane fundamental solutions for traction integral equations}

The fundamental solutions for the traction integral equations are linear combination of the derivatives of the fundamental solution for boundary integral equations; the expressions for the kernels $D_{\alpha \beta \gamma}^{m}$ and $S_{\alpha \beta \gamma}^{m}$ are:

$$
\begin{gathered}
D_{\alpha \beta \gamma}^{m}=\frac{1}{4 \pi r}\left[(1-\nu)\left(\delta_{\gamma \alpha} r_{, \beta}+\delta_{\gamma \beta} r_{, \alpha}-\delta_{\alpha \beta} r_{, \gamma}\right)+2(1+\nu) r_{, \alpha} r_{, \beta} r_{, \gamma}\right] \\
S_{\alpha \beta \gamma}^{m}=\frac{B(1-\nu)}{4 \pi r^{2}}\left\{2 r_{, n}\left[(1-\nu) \delta_{\alpha \beta} r_{, \gamma}+\nu\left(\delta_{\gamma \alpha} r_{, \beta}+\delta_{\gamma \beta} r_{, \alpha}\right)-4(1+\nu) r_{, \alpha} r_{, \beta} r_{, \gamma}\right]+\right. \\
+2 \nu\left(n_{\alpha} r_{, \beta} r_{, \gamma}+n_{\beta} r_{, \alpha} r_{, \gamma}\right)+(1-\nu)\left(2 n_{\gamma} r_{, \alpha} r_{, \beta}+n_{\beta} \delta_{\alpha \gamma}+n_{\alpha} \delta_{\beta \gamma}\right)+ \\
\left.-(1-3 \nu) n_{\gamma} \delta_{\alpha \beta}\right\}
\end{gathered}
$$


where $D_{\alpha \beta \gamma}^{m}$ are strongly singular kernels of order $O(1 / r)$ and $S_{\alpha \beta \gamma}^{m}$ are hyper-singular of the order $O\left(1 / r^{2}\right)$.

\section{Appendix B}

\section{Boundary values}

In order to check the accuracy of the boundary deflection derivatives extrapolation, the simply supported plate under uniform pressure is taken into consideration. The unknown normal tractions at the boundary can be evaluated exactly without considering the large deflection contribution at the boundary. This happens because the actual unknowns are the difference between the real traction and the large deflection additional terms at the boundary. In this way it is possible to solve the problem without the extrapolation of the deflection derivatives at the boundary. By comparing the maximum deflection obtained in this way with the full formulation using different RBF, it is possible to estimate their accuracy. In table (2) the results for several RBF are presented for comparison. It has to be noticed that the shape function derivatives as proposed in [17] has been used as well and it gives the most accurate results for this type of problems, but when applied to more complex geometries the large deflection terms at the boundary may have an irregular behaviour. Thus the RBF are preferred because, although less accurate, they give regular results in all cases where applied.

\begin{tabular}{|c|c|c|c}
\hline \hline$Q_{a \operatorname{dim}}$ & exact & $\sqrt{r^{2}+c^{2}}$ & $1 / \sqrt{r^{2}+c^{2}}$ \\
\hline 50 & 0.934 & 0.930 & 0.936 \\
\hline \hline$Q_{a \operatorname{dim}}$ & $e^{\left(-r^{2} / c^{2}\right)}$ & $\cos (r / b)$ & $1+r+r^{2}+r^{3}$ \\
\hline 50 & 0.937 & 0.9367 & 0.9366 \\
\hline
\end{tabular}

Table 2: Simply supported square plate under large deflection: results for various RBF

All the RBF gave good results but the function $\sqrt{r^{2}+c^{2}} ; c^{2}=2$, was found to be the most accurate and reliable for different cases among the trial functions. Tests on different boundary conditions have been presented in chapter 3 and the results were found satisfactory. The situations where more plates are connected lay in between the free edge and the clamped case, thus the limiting cases have been tried. For the tests 16 DRM points and 80 load steps have been used. 


\section{References}

[1] Aliabadi, M.H., The Boundary Element Method, vol II: application to solids and structures, Chichester, Wiley (2001)

[2] Portela,A, Aliabadi,M.H and Rooke,D.P. The dual boundary element method - effective implementation for crack problems, International journal for numerical methods in engineering, Vol. 33, pp1269-1287 (1992).

[3] Rigby, R.H. and Aliabadi, M.H., Mixed-mode J-integral method for analysis of 3D fracture problems using BEM, Engineering Analysis with Boundary Elements, vol.11, pp.239-256, (1993)

[4] Fung, Y.G., Foundations of Solid Mechanics, Prentice-Hall, Inc., London, (1965)

[5] Huy, C.I., Zehnder A.T. and Potdar, Y.K., Williams meets von Karman: Mode coupling and nonlinearity in the fracture of thin plates, International Journal of Fracture, vol.93, pp.409-429, (1998)

[6] Purbolaksono, J., Aliabadi, M.H., Large deformation of shear deformable plates by the boundary-element method, Journal of Engineering Mathematics, vol.51, pp.211-230, (2005)

[7] Lei, X.-Y., Huang, M.-K. and Wang, X., Geometrically nonlinear analysis of a Reissner type plate by the boundary element method, Computers and Structures, vol.37(6), pp. 911-916, (1990)

[8] Reissner, E., On a variational theorem in elasticity, Journal of Mathematics and Physics, vol. 29, pp. 90-95, (1950)

[9] Tanaka, M., Matsumoto, T. and Zheng, Z.-D., Incremental analysis of finite deflection of elastic plates via boundary-domain-element method, Engineering Analysis with Boundary Elements, vol.17, pp. 123-131, (1996)

[10] Dirgantara, T. and Aliabadi, M. H., A new boundary element formulation for shear deformable shells analysis, International Journal for numerical methods in engineering, vol.45, pp. 1257-1275, (1999)

[11] Dirgantara, T. and Aliabadi, M. H., Boundary element method analysis of assembled plate-structure, Communications in numerical methods in engineering, vol.17, pp. 749-760, (2001)

[12] Dirgantara, T. and Aliabadi, M. H., Crack growth analysis of plates loaded by bending and tension using dual boundary element method, International Journal of Fracture, vol.105 (1), pp.27-47, (2000)

[13] Dirgantara, T. and Aliabadi, M. H., Stress Intensity Factors for cracks in thin plates, Engineering Fracture Mechanics, vol.69, pp. 1465-1486, (2002)

[14] Dirgantara, T. and Aliabadi, M. H., Dual boundary element formulation for fracture mechanics analysis of shear deformable shells,International Journal of Solids and Structures, vol. 38, pp.7769-7800, (2001)

[15] Vander Weeen, F. Application of the boundary integral equation method to Reissner's plate model, International Journal of for Numerical Methods in Engineering, vol. 18, pp. 1-10, (1982)

[16] O.C.Zienkiewicz and R.Taylor, The Finite Element Method, Vol2: Solid Mechanics, B-H, Oxford, (2000)

[17] Wen, P. H., Aliabadi, M. H. and Young, A., Large Deflection Analysis of Reissner's plate by Boundary Element Method, Computer and Structures, vol.83, pp.870-879, (2005) 